\title{
Effective gauge theories of superfluidity with topological order
}

\author{
Yuji Hirono $^{a, b}$ and Yuya Tanizaki ${ }^{c}$ \\ ${ }^{a}$ Asia Pacific Center for Theoretical Physics, \\ Pohang, Gyeongbuk 37673, Korea \\ ${ }^{b}$ Department of Physics, POSTECH, \\ Pohang, Gyeongbuk 37673, Korea \\ ${ }^{c}$ Department of Physics, North Carolina State University, \\ Raleigh, NC 27607, U.S.A. \\ E-mail: yuji.hirono@apctp.org, ytaniza@ncsu.edu
}

ABSTRACT: We discuss the low-energy dynamics of superfluidity with topological order in $(3+1)$ spacetime dimensions. We generalize a topological $B F$ theory by introducing a nonsquare $K$ matrix, and this generalized $B F$ theory can describe massless Nambu-Goldstone bosons and anyonic statistics between vortices and quasiparticles. We discuss the general structure of discrete and continuous higher-form symmetries in this theory, which can be used to classify quantum phases. We describe how to identify the appearance of topological order in such systems and discuss its relation to a mixed 't Hooft anomaly between discrete higher-form symmetries. We apply this framework to the color-flavor locked phase of dense QCD, which shows anyonic particle-vortex statistics while no topological order appears. An explicit example of superfluidity with topological order is discussed.

Keywords: Anyons, Topological Field Theories, Phase Diagram of QCD, Topological States of Matter

ARXiv EPrint: 1904.08570 


\section{Contents}

1 Introduction 1

2 Effective field theory of topologically ordered superfluidity 3

2.1 Effective Lagrangian of general Abelian-Higgs models 3

2.2 Classification of spectra 4

2.3 Non-canonical normalization of gauge fields $\quad 7$

$\begin{array}{lll}\text { 2.3.1 Generalization to non-canonical normalization } & 7\end{array}$

2.3.2 Basis change of gauge fields 8

3 Higher-form symmetries of generalized $B F$ theories $\quad 9$

$\begin{array}{lr}3.1 \text { Continuous higher-form symmetries } & 9\end{array}$

$\begin{array}{lll}3.2 & \text { Discrete higher-form symmetries } & 10\end{array}$

$\begin{array}{lll}3.3 & \text { Particle-vortex statistics } & 11\end{array}$

3.4 Fate of the symmetries and topological order 13

4 Color-flavor locked phase of QCD $\quad \mathbf{1 5}$

$\begin{array}{lll}4.1 \text { Generalized BF theory for CFL phase } & 15\end{array}$

$\begin{array}{lll}4.2 & \text { Emergent higher-form symmetry of CFL phase } & 18\end{array}$

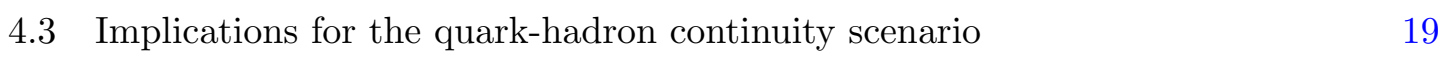

5 Example of superfluidity with topological order $\quad 19$

$\begin{array}{llr}6 & \text { Summary and outlook } & 21\end{array}$

A Moore-Penrose inverse $\quad 22$

B Delta function forms $\quad 22$

C Derivation of the braiding phase (3.13) 23

D Notes regarding basis changes $\quad 24$

D.1 Example 25

D.2 $\mathbb{Z}_{3}$ symmetry action in the original basis $\quad 26$

$\begin{array}{ll}\text { D.3 Calculation in the rotated basis } & 27\end{array}$

$\begin{array}{lll}\text { D.4 More general cases } & 27\end{array}$

\section{Introduction}

Classification of phases of matter has been one of the most fundamental problems in the physics of many-body systems. Different phases of matter have been classified by their symmetries, which led to the theory of spontaneously symmetry breaking [1-4]. It is now realized that quantum phases of matter depends also on "topology", and GinzburgLandau (GL) type classification is not sufficient. An important class of such states is 
called topological order, and there are nontrivial long-range correlation even though no massless excitations exist: topological degeneracy of ground states, anyon statistics of quasiparticles, and so on [5-12]. Low-energy effective description of topological order is given by topological field theories, and the presence of "deconfined" dynamical gauge fields plays an important role for those nontrivial long-range phenomena with mass gap $[13,14]$. Certain classes of topological orders can be understood as a consequences of spontaneous breaking of higher-form (or generalized) global symmetries [15, 16].

One possible direction of further developments on quantum many-body physics is to understand the role of topology in the presence of gapless degrees of freedom. Although we do not have a complete consensus about the definition of topological order in gapless systems, let us temporarily consider it in this paper as a quantum system which has deconfined gauge fields in addition to local gapless excitations. Such theoretical models have been recently studied in the context of quantum criticality of high- $T_{\mathrm{c}}$ cuprates, using gauged GL model [17-20]. There is also an example of such description in the physics of QCD [21-24]. At large baryon densities, QCD matter is expected to exhibit color superconductivity [25-29], which has topological vortices [30-33]. Understanding the role of topology would be important in the discussion of the phase structure of dense nuclear matter $[28,34,35]$ or the possible continuity of vortices between a nuclear superfluid and a color superconductor $[36,37]$. The low-energy theory of a color superconducting phase is described by a topological field theory coupled with massless Nambu-Goldstone (NG) bosons $[34,35,38]$.

Motivated by these recent developments on the possibility of topological order in gapless systems, we study a general framework for studying superfluidity coupled to $B F$-type topological field theory $[8,39-42]$. Starting from a gauged GL model, we derive a dual gauge theory. The effective theory is a generalized $B F$ theory with a non-square $K$ matrix coupled with massless NG bosons. The system is shown to acquire discrete and continuous 2-form/1-form symmetries. As a consequence of the emergent symmetries, the system is shown to exhibit fractional braiding statistics between vortices and quasiparticles. We examine the condition when a topological order appears, which can be also seen as the existence of a mixed 't Hooft anomaly [43] between higher-form symmetries.

This paper is organized as follows. In section 2, we introduce a low-energy effective theory of superfluidity that can also have topological order. In section 3, we identify the continuous and discrete higher-form symmetries of the system and discuss the braiding of quasiparticles and vortices. We also discuss how to detect the topological order in this theory and its interpretation as a mixed 't Hooft anomaly. In section 4, we describe the topological properties of the color-flavor locked phase of dense QCD as an application of the framework. This section is the follow-up of the previous paper [34] with more detailed explanations. In section 5, we discuss an explicit example of superfluidity with topological order. Section 6 is devoted to a summary and outlook. In appendix A, we summarize the properties of the Moore-Penrose inverse. In appendix B, we provide a summary of the properties of delta-function forms. In appendix $\mathrm{C}$, we give a derivation of the braiding phase using the effective theory. Appendix D is devoted to a discussion about the consequence of basis changes. 


\section{Effective field theory of topologically ordered superfluidity}

We aim at describing the low-energy behavior of superfluids with topological order. Here, let us construct a generic low-energy effective theory that consists of $2 \pi$-periodic compact scalar fields and $\mathrm{U}(1)$ gauge fields.

\subsection{Effective Lagrangian of general Abelian-Higgs models}

We consider a $3+1$-dimensional theory with multiple $\mathrm{U}(1)$ symmetries and some parts of them are gauged and couple to dynamical U(1) 1-form gauge fields. We are interested in the low-energy regime of the theory and in this limit, the remaining degrees of freedom are massless modes, that are the NG bosons associated with the spontaneous breaking of $\mathrm{U}(1)$ symmetries. Thus, the system is a superfluid. In addition, there can be topological degrees of freedom.

Let us give a derivation of an effective theory for describing such a system. We take variables $\phi_{i}$, that are (would-be) NG modes associated with the breaking of U(1) symmetries. These are $2 \pi$-periodic scalar fields. They couple to gauge fields through a covariant derivative,

$$
\mathrm{d}_{a} \phi_{i} \equiv \mathrm{d} \phi_{i}+K_{i A} a_{A}
$$

and $a_{A}$ are dynamical Abelian 1-form gauge fields, $a_{A}(x)=\left(a_{A}\right)_{\mu} \mathrm{d} x^{\mu}(A=1, \cdots,|A|)$, and $K_{i A}$ is a $|i| \times|A|,{ }^{1}$ integer-valued matrix. Let us call $a_{A}(x)$ as photons. The covariant derivative is invariant under the 0 -form gauge transformation,

$$
\phi_{i} \mapsto \phi_{i}-K_{i A} \lambda_{A}, \quad a_{A} \mapsto a_{A}+\mathrm{d} \lambda_{A},
$$

where gauge parameters $\lambda_{A}$ are also $2 \pi$-periodic scalars. Because of this interaction, a part of the would-be NG modes are Higgsed. We start with an action,

$$
S=\frac{1}{2} H_{i j} \int \mathrm{d}_{a} \phi_{i} \wedge \star \mathrm{d}_{a} \phi_{j}+\frac{1}{2} G_{A B}^{a} \int \mathrm{d} a_{A} \wedge \star \mathrm{d} a_{B} .
$$

The positivity of the kinetic terms require that $H$ and $G^{a}$ are positive-definite real symmetric matrices.

We shall take an Abelian dual of this theory [44]. The action can be rewritten by introducing $\mathbb{R}$-valued 3 -form fields $h_{i}$ as

$$
S=\frac{1}{8 \pi^{2}} H_{i j}^{-1} \int h_{i} \wedge \star h_{j}-\frac{\mathrm{i}}{2 \pi} \int h_{i} \wedge \mathrm{d}_{a} \phi_{i}+\frac{1}{2} G_{A B}^{a} \int \mathrm{d} a_{A} \wedge \star \mathrm{d} a_{B} .
$$

Solving the equation of motion (EOM) for $h_{i}$, we obtain $h_{i}=2 \pi \mathrm{i} H_{i j} \star \mathrm{d}_{a} \phi_{j}$ and get the original action (2.3). Solving the EOM for $\phi_{i}$, instead, we find $\mathrm{d} h_{i}=0$, and it can be solved as

$$
h_{i}=\mathrm{d} b_{i},
$$

\footnotetext{
${ }^{1}$ We use the notation to represent the number of rows (or columns) of a matrix by the absolute value of the index.
} 
where $b_{i}(x)=\frac{1}{2}\left(b_{i}\right)_{\mu \nu} d x^{\mu} \wedge d x^{\nu}(i=1, \cdots,|i|)$ are 2-form $\mathrm{U}(1)$ gauge fields. ${ }^{2}$ Plugging this into $h_{i}$, the action is rewritten as

$$
S=\frac{1}{2} G_{i j}^{b} \int \mathrm{d} b_{i} \wedge \star \mathrm{d} b_{j}+\frac{1}{2} G_{A B}^{a} \int \mathrm{d} a_{A} \wedge \star \mathrm{d} a_{B}+\mathrm{i} \frac{K_{i A}}{2 \pi} \int b_{i} \wedge \mathrm{d} a_{A},
$$

where we introduce $G_{i j}^{b} \equiv H_{i j}^{-1} / 4 \pi^{2}$. The first two terms are the usual kinetic terms, and the last term is the topological $B F$ term. $^{3}$ Physical observables can be calculated by the partition function,

$$
Z=\int \mathcal{D} a \mathcal{D} b \mathrm{e}^{-S[a, b]} .
$$

In this path integral, we sum over all possible gauge fields, satisfying the canonical Dirac quantization conditions,

$$
\int_{S} \mathrm{~d} a_{A} \in 2 \pi \mathbb{Z}, \int_{V} \mathrm{~d} b_{i} \in 2 \pi \mathbb{Z},
$$

for each closed 2-submanifold $S$ and 3-submanifold $V$ of the spacetime.

Physical operators that we will focus on are Wilson loop operators and vortex operators,

$$
W_{A}(C)=\exp \left(\mathrm{i} \int_{C} a_{A}\right), \quad V_{i}(S)=\exp \left(\mathrm{i} \int_{S} b_{i}\right)
$$

where $C$ is a world-line of a test particle, and $S$ is a vortex world-sheet.

\subsection{Classification of spectra}

Since we are interested in the low-energy physics, we only retain massless modes and topological sector. Before studying the global nature of the theory, let us clarify its local dynamics to identify the massless sector of $b$ and $a$. For this purpose, let us write down the EOMs of $b_{i}$ and $a_{A}$ :

$$
\begin{aligned}
G_{i j}^{b} \mathrm{~d} \star \mathrm{d} b_{j}-\frac{\mathrm{i}}{2 \pi} K_{i A} \mathrm{~d} a_{A} & =0, \\
G_{A B}^{a} \mathrm{~d} \star \mathrm{d} a_{B}+\frac{\mathrm{i}}{2 \pi} K_{i A} \mathrm{~d} b_{i} & =0 .
\end{aligned}
$$

Combining these EOMs, we find that $\mathrm{d} b_{i}$ must satisfy

$$
\left(G^{b} \Delta+\frac{1}{4 \pi^{2}} K\left(G^{a}\right)^{-1} K^{T}\right) \mathrm{d} b=0,
$$

and that $\mathrm{d} a_{A}$ must satisfy

$$
\left(G^{a} \Delta+\frac{1}{4 \pi^{2}} K^{T}\left(G^{b}\right)^{-1} K\right) \mathrm{d} a=0 .
$$

\footnotetext{
${ }^{2}$ This normalization is determined by the global structure: let us set our spacetime as 4-torus $T^{4}$ of size $L$ as an example, then the $2 \pi$-periodic scalar $\phi$ can be decomposed as $\phi=\frac{2 \pi}{L} n_{\mu} x^{\mu}+\tilde{\phi}$, where $n_{\mu} \in \mathbb{Z}$ and $\tilde{\phi}$ is the $\mathbb{R}$-valued field. The above $\mathrm{EOM}, \mathrm{d} h=0$, comes out of the path integral over $\tilde{\phi}$. The summation over $\left\{n_{\mu}\right\} \in \mathbb{Z}^{4}$ further requires that $\int_{T^{3}} h \in 2 \pi \mathbb{Z}$ for each 3 -torus and we find the correct normalization (2.5).

${ }^{3}$ One could consider further generalization by adding $a_{A} \wedge a_{B} \wedge \mathrm{d} a_{C}, b_{i} \wedge b_{j}$, etc., with appropriate coefficients and modification of gauge transformations, like a twist term of Dijkgraaf-Witten theory [13, 45, 46]. In this paper, however, we do not pursue along this direction.
} 
Here, $\Delta=\mathrm{d} \delta+\delta \mathrm{d}$ is the form Laplacian with the codifferential $\delta=-\star \mathrm{d} \star$, and $K^{T}$ represents the transpose of $K$ matrix. Therefore, the mass matrix $M^{b}$ of $b_{i}$ is given by

$$
\left(M^{b}\right)^{2}=\frac{1}{4 \pi^{2}}\left(G^{b}\right)^{-1 / 2} K\left(G^{a}\right)^{-1} K^{T}\left(G^{b}\right)^{-1 / 2},
$$

and the mass matrix $M^{a}$ of $a_{A}$ is given by

$$
\left(M^{a}\right)^{2}=\frac{1}{4 \pi^{2}}\left(G^{a}\right)^{-1 / 2} K^{T}\left(G^{b}\right)^{-1} K\left(G^{a}\right)^{-1 / 2} .
$$

Here, we note that squared roots of $G^{a}$ and $G^{b}$ are well defined since they are positive matrix.

Let us discuss how massless degrees of freedom depend on the structure of the $K$ matrix. When $|i|=|A|$ (i.e. $K$ is a square matrix) and $\operatorname{det} K \neq 0$, all the particles get nonzero mass because neither $M^{b}$ nor $M^{a}$ have zero eigenvalues, and the $B F$ theory for superconductivity is reproduced. We are interested in the situation where superfluidity is present. In this case, there exists at least one massless NG modes, which is realized when $\operatorname{dim}(\operatorname{coker} K) \neq 0$. Indeed, for each vector $\boldsymbol{D}^{\bar{\alpha}} \in \operatorname{coker} K$, i.e.

$$
\left(\boldsymbol{D}^{\bar{\alpha}}\right)^{T} \cdot K=0
$$

we can find the null eigenvector of the mass matrix:

$$
\left(M^{b}\right)^{2} \sqrt{G^{b}} \boldsymbol{D}^{\bar{\alpha}}=0 .
$$

Since $K$ is in general a non-square matrix, there always exist massless NG modes if $|i|>|A|$.

There can also be remaining massless photons, when $\operatorname{dim}(\operatorname{ker} K) \neq 0$. We denote the basis of the kernel and cokernel as $\boldsymbol{C}^{\alpha} \in \operatorname{ker} K$, and $\boldsymbol{D}^{\bar{\alpha}} \in$ coker $K$, namely, they satisfy

$$
K_{i A} C_{A}^{\alpha}=0, \quad D_{i}^{\bar{\alpha}} K_{i A}=0 .
$$

We can identify the massless NG modes and massless photons as

$$
b_{0} \in \operatorname{coker} K, \quad a_{0} \in \operatorname{ker} K,
$$

because $a_{0}$ and $b_{0}$ does appear in the $B F$ term. The numbers of massless NG modes and massless photons, $|\bar{\alpha}|$ and $|\alpha|$, are given by the dimensions of the cokernel and kernel of $K$, respectively:

$$
|\bar{\alpha}|=\operatorname{dim}(\operatorname{coker} K), \quad|\alpha|=\operatorname{dim}(\operatorname{ker} K) .
$$

Those massless modes can be identified by projection matrices,

$$
\left(a_{0}\right)_{A}=P_{A B}^{a} a_{B}, \quad\left(b_{0}\right)_{i}=P_{i j}^{b} b_{j},
$$

where $P^{a}$ and $P^{b}$ are orthogonal projectors to the kernel and cokernel of $K$. They can be expressed using the Moore-Penrose inverse ${ }^{4} K^{+}$of the $K$ matrix, which is a generalization

\footnotetext{
${ }^{4}$ We summarize the properties of the Moore-Penrose inverse in appendix A.
} 
of a matrix inverse. Given an arbitrary matrix, the Moore-Penrose inverse always exists and is unique. The projectors are given by ${ }^{5}$

$$
P_{A B}^{a}=\delta_{A B}-\left[K^{+} K\right]_{A B}, \quad P_{i j}^{b}=\delta_{i j}-\left[K K^{+}\right]_{i j} .
$$

Using this orthonormal projection, we denote the gauge fields as

$$
a=a_{0}+a_{\perp}, \quad b=b_{0}+b_{\perp} .
$$

This decomposition diagonalizes the mass term, but it does not necessarily diagonalize the kinetic term: for example, the kinetic term of $b$ becomes

$$
\frac{1}{2} \int \mathrm{d} b^{T} \wedge G^{b} \star \mathrm{d} b=\frac{1}{2} \int\left(\mathrm{d} b_{0}^{T} \wedge G^{b} \star \mathrm{d} b_{0}+2 \mathrm{~d} b_{\perp}^{T} \wedge G^{b} \star \mathrm{d} b_{0}+\mathrm{d} b_{\perp}^{T} \wedge G^{b} \star \mathrm{d} b_{\perp}\right) .
$$

In the low-energy limit, the last term can be neglected since it only describes the exponential decay of massive excitations, and one should retain the first and the second terms. The mixed kinetic term $\mathrm{d} b_{\perp}^{T} \wedge G^{b} \star \mathrm{d} b_{0}$ vanishes identically if and only if

$$
P^{b} G^{b}\left(1-P^{b}\right)=0
$$

Since $G^{b}$ and $P^{b}$ are both symmetric matrices, this condition is equivalent to

$$
\left[G^{b}, P^{b}\right]=0
$$

We obtain the same conclusion also for the photon fields $a$. In the rest of this paper, we assume that

$$
\left[G^{a}, P^{a}\right]=0, \quad\left[G^{b}, P^{b}\right]=0,
$$

so that the mixed kinetic terms between massless and heavy modes vanish identically. ${ }^{6}$ In concrete examples, the condition (2.26) may be implied by a certain symmetry of the UV theory. We expect that this condition is important to protect the topological order under the existence of gapless excitations. As a consequence of assumption (2.26), we obtain the low-energy effective action as

$$
S_{\text {eff }}=\mathrm{i} \frac{K_{i A}}{2 \pi} \int b_{i} \wedge \mathrm{d} a_{A}+\frac{1}{2} G_{i j}^{b} \int \mathrm{d}\left(b_{0}\right)_{i} \wedge \star \mathrm{d}\left(b_{0}\right)_{j}+\frac{1}{2} G_{A B}^{a} \int \mathrm{d}\left(a_{0}\right)_{A} \wedge \star \mathrm{d}\left(a_{0}\right)_{B},
$$

where $b_{0}$ and $a_{0}$ are massless contributions as identified above. We call this as the generalized $B F$ theory.

This action (2.27) can describe various physical situations, depending on the choice of $K$ matrices. Possible physical situations are classified according to the numbers, $|\bar{\alpha}|,|\alpha|,|i|$, and $|A|$. Note that, according to Fredholm's index theorem, those numbers are related as

$$
|\bar{\alpha}|-|\alpha|=|i|-|A| .
$$

\footnotetext{
${ }^{5}$ We use the notation where contracted matrix indices may be omitted when there is no confusion, for example, $\left[K^{+} K\right]_{A B}=K_{A i}^{+} K_{i B}$.

${ }^{6}$ In appendix D, we discuss the consequence of the mixed kinetic terms.
} 
Based on this relation, possible situations of (2.27) can be classified as follows:

- $|\bar{\alpha}|=|\alpha|=0,|i|=|A|$ : all the excitations are massive. In this case, The $K$ matrix is square and regular, which corresponds to $B F$ theoretical description of superconductors.

- $|\alpha|=|A|=0,|\bar{\alpha}|=|i|$ : superfluids with no topological order.

- $|\bar{\alpha}|=|i|=0,|\alpha|=|A|$ : pure Maxwell theory.

- In other cases, superfluidity and topological order may coexist.

\subsection{Non-canonical normalization of gauge fields}

\subsubsection{Generalization to non-canonical normalization}

So far, we are working on the theory (2.6) with the canonically normalized gauge fields $b_{i}$ and $a_{A}$ as in (2.8). Instead, we can work on more general normalization of these gauge fields, and let us discuss such cases in this section. The motivation for this generalization is that gauge fields of the low-energy effective theory can be emergent and is not necessarily ensured to be canonically normalized when we derive it from the UV theory. Therefore, it is important to establish the way to analyze such cases. The normalization of gauge fields does not affect local dynamics, and thus the discussion in section 2.2 is unaffected while the global nature of the theory can be changed drastically.

The normalization of gauge fields is related to the choice of physically observable Wilson loops and vortex operators. Let us replace (2.8) by a generic normalization condition (Dirac quantization condition) for $a_{A}$ and $b_{i}$ as

$$
Q_{A B} \int_{S} \mathrm{~d} a_{B} \in 2 \pi \mathbb{Z}, \quad \int_{V} \mathrm{~d} b_{j} R_{j i} \in 2 \pi \mathbb{Z}
$$

where $S$ and $V$ are 2D and 3D subspace without boundary, and $Q_{A B}$ and $R_{i j}$ are invertible matrices with integer elements. Correspondingly, the matrices $Q$ and $R$ specify the set of generators of gauge-invariant Wilson loops and vortex operators,

$$
W_{A}^{(Q)} \equiv \exp \left(\mathrm{i} Q_{A B} \int_{C} a_{B}\right), \quad V_{i}^{(R)} \equiv \exp \left(\mathrm{i} \int_{S} b_{j} R_{j i}\right)
$$

The naive Wilson loop and surface operators (2.9) are no longer gauge invariant in this normalization.

Let us discuss the gauge redundancy of the action. It is invariant under 0-form and 1-form gauge transformations,

$$
a_{A} \mapsto a_{A}+Q_{A B}^{-1} \mathrm{~d} \lambda_{B}, \quad b_{i} \mapsto b_{i}+R_{j i}^{-1} \mathrm{~d} \lambda_{j},
$$

where $\lambda_{A}$ is a $2 \pi$-periodic scalar and $\lambda_{i}$ is a $\mathrm{U}(1) 1$-form fields. They satisfy

$$
\int \mathrm{d} \lambda_{A} \in 2 \pi \mathbb{Z}, \quad \int \mathrm{d} \lambda_{i} \in 2 \pi \mathbb{Z},
$$


where the integrations are over closed submanifolds of corresponding dimensions. Introducing the matrices $Q^{-1}$ and $R^{-1}$ is necessary for the consistency with the normalization condition (2.29). The variation of the action is

$$
\delta_{1} S_{\text {eff }}=\frac{K_{i A} Q_{A B}^{-1}}{2 \pi} \int \mathrm{d} b_{i} \wedge \mathrm{d} \lambda_{B}, \quad \delta_{2} S_{\text {eff }}=\frac{R_{j i}^{-1} K_{i A}}{2 \pi} \int \mathrm{d} \lambda_{i} \wedge \mathrm{d} a_{A} .
$$

Note that the integration gives

$$
\int \mathrm{d} b_{i} \wedge \mathrm{d} \lambda_{B}=(2 \pi)^{2} R_{j i}^{-1} n_{j} m_{B}, \quad \int \mathrm{d} \lambda_{i} \wedge \mathrm{d} a_{A}=(2 \pi)^{2} Q_{A B}^{-1} m_{B}^{\prime} n_{i}^{\prime}
$$

where $n_{i}, n_{i}^{\prime}, m_{B}, m_{B}^{\prime}$ are integer vectors. The change of the action under 1 -form and 2 -form gauge transformation is now given by

$$
\delta_{1} S_{\text {eff }}=2 \pi n^{T} R^{-1} K Q^{-1} m, \quad \delta_{2} S_{\text {eff }}=2 \pi n^{\prime T} R^{-1} K Q^{-1} m^{\prime}
$$

Thus, the gauge invariance requires that the $K$ matrix should be chosen so that the each element of the matrix $\left[R^{-1} K Q^{-1}\right]_{i A}$ is an integer. This reproduces the fact that $K$ should be an integer-valued matrix when we take $Q=1$ and $R=1$, i.e., the canonical normalization (2.8) of gauge fields.

\subsubsection{Basis change of gauge fields}

As we have shown, a theory can be specified by a set of integer-valued matrices $(K, Q, R)$. However, not every theory associated with a set $(K, Q, R)$ describes a distinct system. This ambiguity was discussed in ref. [14] in the case of the topological $B F$ theory for Abelian anyons, and we extend it to the case of our generalized setup.

We may work on another basis of gauge fields $\widetilde{a}_{A}, \widetilde{b}_{i}$, which are related to the original fields as

$$
a_{A}=M_{A B} \widetilde{a}_{B}, \quad b_{i}=\widetilde{b}_{j} N_{j i}
$$

with some invertible matrices $M$ and $N$. Under the basis change, the $K$ matrix should be replaced as

$$
\widetilde{K}=N K M
$$

One should not forget that, together with eq. (2.36), we also have to transform the charge matrices,

$$
\widetilde{Q}=Q M, \quad \widetilde{R}=N R .
$$

Under this change of normalization, the coefficients of kinetic terms are also changed as

$$
\widetilde{G}^{b}=N G^{b} N^{T}, \widetilde{G}^{a}=M^{T} G^{a} M .
$$

The theories connected by this transformation are equivalent and give the same physical results. For example, gauge invariance of the theory is not affected by the basis change, since the factor $R^{-1} K Q^{-1}$ appearing in eq. (2.35) is invariant under the basis change, eq. (2.36):

$$
\widetilde{R}^{-1} \widetilde{K} \widetilde{Q}^{-1}=R^{-1} K Q^{-1}
$$


One should be cautious in changing the basis because it in general introduce the coupling between massive and massless gauge fields in the kinetic terms, which complicates the analysis. One may seek a simpler expression of $K$ matrix by changing the basis; ${ }^{7}$ for example, we can always obtain one of the simplest expressions for $K$ matrix by considering the Smith normal form ${ }^{8}$

$$
K=U^{-1} K^{\prime} V^{-1},
$$

where $U$ and $V$ are integer matrices with $\operatorname{det}(U)=\operatorname{det}(V)= \pm 1$ and $K^{\prime}$ takes the form

$$
K^{\prime}=\left(\begin{array}{cccc}
d_{1} & 0 & 0 & \ldots \\
0 & d_{2} & 0 & \ldots \\
0 & 0 & \ddots & \\
\vdots & \vdots & &
\end{array}\right)
$$

where $d_{i}$ are also integers. Since $\operatorname{det}(U)=\operatorname{det}(V)= \pm 1$, we can stay in the canonical normalization of gauge fields when the original expression is in the canonical normalization. If (2.26) is maintained, this is the simplest basis to work on. However, this is not always the case. The theories considered in sections 4 and 5 are such examples.

Generically, if the basis change matrices satisfy $N N^{T}=1$ and $M^{T} M=1$, the condition (2.26) is maintained under the basis change. In this case, the Moore-Penrose inverses of old and new $K$ matrices are related by ${ }^{9}$

$$
\widetilde{K}^{+}=M^{-1} K^{+} N^{-1} \text {. }
$$

Otherwise, the condition (2.26) is not kept and eq. (2.43) does not hold. In such cases, the change of basis introduces the coupling between massless and massive modes, which complicates the evaluation of correlation functions and identification of symmetry generators. For a detailed discussion on the effects of the coupling of massless and massive modes, see appendix D.

\section{Higher-form symmetries of generalized $B F$ theories}

Let us examine the global higher-form symmetry of the action (2.27). Depending on the structure of the $K$ matrix, there can exist discrete 1-form and 2-form symmetries. In the presence of massless phonons and photons, there are also continuous 1-form and 2-form symmetries. Here let us identify those symmetries.

\subsection{Continuous higher-form symmetries}

The action (2.27) has continuous (or U(1)) 1-form or 2-form global symmetries in the presence of massless photons or NG bosons, respectively. The symmetry transformation is given by

$$
a_{A} \mapsto a_{A}+\epsilon C_{A}^{\alpha} \mu_{\alpha}, \quad b_{i} \mapsto b_{i}+\epsilon D_{i}^{\bar{\alpha}} \rho_{\bar{\alpha}},
$$

\footnotetext{
${ }^{7}$ A similar comment is true also for 3d Chern-Simons theory with a slightly different reasoning [14].

${ }^{8}$ Authors appreciate the anonymous referee for pointing this out.

${ }^{9}$ When $K$ is invertible, eq. (2.43) is trivially true.
} 
where $\mu_{\alpha}$ and $\rho_{\bar{\alpha}}$ are flat 1-form and 2-form fields and $\epsilon$ is an infinitesimal parameter. One can immediately see that the effective action (2.27) is invariant under the transformation (3.1), using $K_{i A} C_{A}^{\alpha}=0$ and $D_{i}^{\bar{\alpha}} K_{i A}=0$. The number of U(1) 1-form symmetry is given by $|\alpha|=\operatorname{dim}(\operatorname{ker} K)$, and the number of $\mathrm{U}(1) 2$-form symmetries is $|\bar{\alpha}|=\operatorname{dim}(\operatorname{coker} K)$. They are the same as the number of massless photons and massless NG modes. Those symmetry act on Wilson loops and vortex operators as a phase rotation,

$$
W_{A} \mapsto W_{A} \exp \left(2 \pi \mathrm{i} \epsilon C_{A}^{\alpha} \mu_{\alpha}\right), \quad V_{i} \mapsto V_{i} \exp \left(2 \pi \mathrm{i} \epsilon D_{i}^{\bar{\alpha}} \rho_{\bar{\alpha}}\right) .
$$

\subsection{Discrete higher-form symmetries}

The theory can have discrete higher-form symmetries. Consider the following transformation of the 2-form gauge field $b_{i}$,

$$
b_{i}^{T} \mapsto b_{i}^{T}+\left[q^{T} Q K^{+}\right]_{i} \lambda,
$$

where $\lambda=\lambda(x)$ is a closed 2-form connection, i.e. $\mathrm{d} \lambda=0$, with quantized holonomy $\int_{S} \lambda \in 2 \pi \mathbb{Z}$ over a closed surface $S$, and $q_{A}$ is a charge vector. In order to make a one-toone correspondence between $q_{A}$ and this transformation, we require that the charge vector has to satisfy

$$
q^{T} \cdot Q \in\left(\operatorname{coker} K^{+}\right)^{\perp}=(\operatorname{ker} K)^{\perp} .
$$

The kinetic term for $b_{0}$ is invariant under (3.3), since it does not shift massless NG modes $\left(\delta\left[P_{i j}^{b} b_{i}\right]=0\right.$ follows from the definition of the Moore-Penrose inverse). The variation of the action is

$$
\delta_{q} S_{\text {eff }}=\frac{\mathrm{i}}{2 \pi}\left[q^{T} Q K^{+} K\right]_{A B} \int \lambda \wedge \mathrm{d} a_{B}
$$

Noting that $K^{+} K$ is the projection matrix to $(\operatorname{ker} K)^{\perp}$,

$$
\left(q^{T} Q\right)_{A}\left[K^{+} K\right]_{A B}=\left(q^{T} Q\right)_{B}
$$

Thus,

$$
\delta_{q} S_{\text {eff }}=2 \pi \mathrm{i}\left(q^{T} Q\right)_{A} \int \frac{\lambda}{2 \pi} \wedge \frac{\mathrm{d} a_{A}}{2 \pi} \in \sum_{A} 2 \pi \mathrm{i} q_{A} \mathbb{Z},
$$

where we used the normalization condition for $a_{A}$. In order for this to generate symmetry, we must require $\delta_{q} S_{\text {eff }} \in 2 \pi \mathrm{i} \mathbb{Z}$, and then $q_{A}$ has to be an integer-valued vector.

Another necessary requirement of symmetry is that there must exist a physical operator with nontrivial transformation. In this case, if vortex operators in (2.30) transforms nontrivially under (3.3), it is a 2 -form symmetry of the system. The action of the discrete 2 -form transformation (3.3) is given by a phase rotation,

$$
V_{i}^{(R)} \mapsto V_{i}^{(R)} \exp \left(2 \pi \mathrm{i}\left[q^{T} Q K^{+} R\right]_{i}\right) .
$$

If there exists a charge vector $q$ with $q^{T} Q \in\left(\text { coker } K^{+}\right)^{\perp}$ such that the transformation (3.8) is nontrivial, then the system has a discrete 2 -form symmetry.

Similarly, the discrete 1-form symmetry transformation is given by

$$
a_{A} \mapsto a_{A}+\left[K^{+} R p\right]_{A} \omega,
$$


where $\omega$ is a flat connection with $\int \omega \in 2 \pi \mathbb{Z}$, and $R p \in(\operatorname{coker} K)^{\perp}$. The action is varied as

$$
\delta_{p} S_{\text {eff }}=\frac{\mathrm{i}}{2 \pi}\left[K K^{+} R p\right]_{i} \int \mathrm{d} b_{i} \wedge \omega .
$$

Noting that $K K^{+} R p=R p$, we have $\delta S_{\text {eff }} \in 2 \pi \mathrm{i} \mathbb{Z}$ when $p$ is an integer vector, and the system is invariant under the 1-form transformation. On the gauge-invariant Wilson loops in $(2.30)$, it acts as

$$
W_{A}^{(Q)} \mapsto W_{A}^{(Q)} \exp \left(2 \pi \mathrm{i}\left[Q K^{+} R p\right]_{A}\right) .
$$

If there is a charge vector $p_{i}$ such that eq. (3.11) is a nontrivial transformation, this is a discrete 1-form symmetry.

\subsection{Particle-vortex statistics}

Let us discuss the nature of particle-vortex statistics. Test particle of charge $q$ and vortex with charge $p$ are represented by

$$
W_{q}^{(Q)}(C)=\exp \left(\mathrm{i}\left(q^{T} Q\right)_{A} \int_{C} a_{A}\right), \quad V_{p}^{(R)}(S)=\exp \left(\mathrm{i} \int_{S} b_{i}(R p)_{i}\right),
$$

respectively, and the gauge invariance requires that $q$ and $p$ are integer-valued vectors. Here, $C$ denotes the world-line of the test particle $q$, and $S$ denotes the world-sheet of the vortex $p$. As shown in appendix $\mathrm{C}$, the correlation function of these operators in the effective theory (2.27) satisfies $^{10}$

$$
\frac{\left\langle W_{q}^{(Q)}(C) V_{p}^{(R)}(S)\right\rangle}{\left\langle W_{q}^{(Q)}(C)\right\rangle\left\langle V_{p}^{(R)}(S)\right\rangle}=\exp \left[-2 \pi \mathrm{i}\left(q^{T} Q K^{+} R p\right) \operatorname{Lk}(S, C)\right],
$$

where $\operatorname{Lk}(S, C)$ is the linking number of the loop $C$ and the surface $S$. This phase $\mathrm{e}^{2 \pi \mathrm{i}\left(q^{T} Q K^{+} R p\right)}$ gives the mutual statistics between test particles and vortices. Here, we intensionally use the same symbol $q, p$ to represent the charges of $W, V$ and to parametrize the discrete higher-form transformations in section 3.2. We shall soon see that the discrete higher-form symmetries are generated by these operators when $p, q$ are chosen so that $V_{p}^{(R)}$, $W_{q}^{(Q)}$ are topological [15].

In order to identify the generators of discrete higher-form symmetries, let us discuss when the vortex operator $V_{p}$ becomes topological. This can be seen by deforming the surface as

$$
\begin{aligned}
V_{p}^{(R)}(S+\delta S) & =V_{p}^{(R)}(S) \exp \left(\mathrm{i}(R p)_{i}\left\{\int_{S+\delta S} b_{i}-\int_{S} b_{i}\right\}\right) \\
& =V_{p}^{(R)}(S) \exp \left(\mathrm{i}(R p)_{i} \int_{V} \mathrm{~d} b_{i}\right)
\end{aligned}
$$

\footnotetext{
${ }^{10}$ When the orthogonality condition is not satisfied and there are mixed kinetic terms of massless and massive modes, there will be corrections, as explained in appendix D. Compare this equation with eq. (D.38).
} 
where $V$ is the volume swept by the continuous deformation $S \rightarrow S+\delta S$, or $\delta S=\partial V$. Therefore, the operator $V_{p}^{(R)}$ is topological, i.e. $V_{p}^{(R)}(S+\delta S)=V_{p}^{(R)}(S)$, if

$$
(R p)_{i} \mathrm{~d} b_{i}=0
$$

by using the EOM of (2.27). This happens when the vortex decouples from the massless phonon, i.e. $R p \in(\operatorname{coker} K)^{\perp}$. Indeed, the EOM of (2.27) with respect to $a_{A}$ gives

$$
\left(\mathrm{d} \star \mathrm{d} a^{T}\right) G^{a}\left(1-K^{+} K\right)+\frac{\mathrm{i}}{2 \pi} \mathrm{d} b^{T} K=0 .
$$

Multiplying $K^{+} R p$ from the right and using $K K^{+} R p=R p$ for $R p \in(\operatorname{coker} K)^{\perp}$, we get (3.15). Other vortex operators with $R p \notin$ (coker $K)^{\perp}$ show non-topological behaviors because of its coupling to massless NG excitations. Let us now discuss the physical meaning of the expectation value (3.13) when we consider the topological surface operator $V_{p}^{(R)}$ with $R p \in(\text { coker } K)^{\perp}$. Taking $S$ as a two sphere singly linked to $C$, we get

$$
\left\langle W_{q}^{(Q)}(C) V_{p}^{(R)}(S)\right\rangle=\mathrm{e}^{2 \pi \mathrm{i}\left(q^{T} Q K^{+} R p\right)}\left\langle W_{q}(C)\right\rangle,
$$

because $\left\langle V_{p}^{(R)}(S)\right\rangle=1$ as $S$ can be shrunk to a point. This explicitly shows that the insertion of $V_{p}^{(R)}$ with $R p \in(\operatorname{coker} K)^{\perp}$ is nothing but the 1-form transformation (3.9).

The same discussion applies for the Wilson loop. When $q^{T} Q \notin(\operatorname{ker} K)^{\perp}$, the Wilson loop obeys Coulomb law. $W_{q}^{(Q)}(C)$ shows topological dependence on $C$ if and only if

$$
\left(q^{T} Q\right)_{A} \mathrm{~d} a_{A}=0
$$

by the EOM of (2.27), which is equivalent to the decoupling condition to massless photons, i.e. $q^{T} Q \in(\operatorname{ker} K)^{\perp}$. When $q^{T} Q \in(\operatorname{ker} K)^{\perp}$, the expectation value (3.13) can be written as

$$
\left\langle W_{q}^{(Q)}(C) V_{p}^{(R)}(S)\right\rangle=\mathrm{e}^{2 \pi \mathrm{i}\left(q^{T} Q K^{+} R p\right)}\left\langle V_{p}^{(R)}(S)\right\rangle
$$

because $\left\langle W_{q}^{(Q)}(C)\right\rangle=1$ by its topological nature. This is nothing but the 2 -form transformation (3.3) if the phase $\mathrm{e}^{2 \pi \mathrm{i}\left(q^{T} Q K^{+} R p\right)}$ is nontrivial for some vortex charge $p$.

Note that the braiding phase $\mathrm{e}^{\mathrm{i}\left(q^{T} Q K^{+} R p\right)}$ is invariant under

$$
(R p)_{i} \mapsto(R p)_{i}+[K \Lambda]_{i}
$$

where $\Lambda_{A} \in(\operatorname{ker} K)^{\perp}$ is an integer vector. Two charge vectors related by this relation are topologically equivalent. Likewise, $\mathrm{e}^{\mathrm{i}\left(q^{T} Q K^{+} R p\right)}$ is invariant under

$$
\left(q^{T} Q\right)_{A} \mapsto\left(q^{T} Q\right)_{A}+\left[\left(\Lambda^{\prime}\right)^{T} K\right]_{A},
$$

with an integer vector $\Lambda_{i}^{\prime} \in(\operatorname{coker} K)^{\perp}$. 


\subsection{Fate of the symmetries and topological order}

Let us discuss the fate of symmetries. For notational simplicity, let us consider the case $Q=1$ and $R=1$ in this subsection. The result for general cases can be reproduced by replacing $\left(q^{T} K^{+} p\right)$ by $\left(q^{T} Q K^{+} R p\right)$.

When discrete higher-form symmetries are spontaneously broken, there appear deconfined anyons and the system acquires a topological order. Let us diagnose the existence of topological order in the current theory. The charged object of a discrete 2-form symmetry is a vortex operator with a vortex charge $p$. Existence of a discrete 2-form symmetry indicates that there exists a topological Wilson loop. A Wilson loop with charge $q \in(\text { ker } K)^{\perp}$ induces a 2-form transformation on a vortex of the form,

$$
V_{p} \mapsto V_{p} \exp \left[2 \pi \mathrm{i} q^{T} K^{+} p\right] .
$$

A discrete 2-form symmetry is spontaneously broken if the expectation value of the vortex operator obeys the surface law at large vortex world-surface $S$,

$$
\left.\left\langle V_{p}(S)\right\rangle \simeq \exp (-\kappa \text { perimeter }[S])\right) .
$$

If a vortex operator is charged under a $\mathrm{U}(1) 2$-form symmetry, $\left\langle V_{p}(S)\right\rangle$ decays faster than the surface law, meaning that the 2 -form symmetry is unbroken. This is because, in 4 spacetime dimensions, 2 -form continuous symmetry cannot be broken due to the generalized Coleman-Mermin-Wagner theorem for higher-form symmetry [15, 47]. Physically, this corresponds to the fact that vortices couple to massless NG bosons and feel logarithmic confining force. In order for the system to have a topological order, there has to be an excitation that is neutral under U(1) 1-form or 2-form symmetries. A U(1) 2-form transformation (3.1) induces a phase rotation of the form

$$
V_{p} \mapsto V_{p} \exp \left[2 \pi \mathrm{i}\left(D_{i}^{\bar{\alpha}} \epsilon_{\bar{\alpha}}\right) p_{i}\right]
$$

where $\epsilon_{\bar{\alpha}}$ is a continuous parameter. In order for a vortex to be neutral under this symmetry, we have to take the vortex charge as $p \in(\operatorname{coker} K)^{\perp}$, which is the same condition with the one that $V_{p}(S)$ is a topological surface operator. If there is a generator of discrete 2 -form symmetry specified by $q$ that acts nontrivially on such vortices, those vortices are deconfined and they generate 1-form symmetries. Then the system can have a pair of broken 1-form and 2-form symmetries specified by the pair $(p, q)$. In such a case, the system is topologically ordered.

To summarize the argument above, appearance of topological order in the theory $(2.27)$ can be detected by the following condition:

a There exists a pair of integer vectors $(p, q) \in(\operatorname{coker} K)^{\perp} \times(\operatorname{ker} K)^{\perp}$, such that $\mathrm{e}^{2 \pi \mathrm{i}\left(q^{T} K^{+} p\right)} \neq 1 .^{11}$

\footnotetext{
${ }^{11}$ One might think that the former condition $(p, q) \in(\operatorname{coker} K)^{\perp} \times(\operatorname{ker} K)^{\perp}$ is redundant, because the components in coker $K$ or ker $K$ are projected out when they are contracted with $K^{+}$and we can just project $p$ or $q$ to $(\text { coker } K)^{\perp}$ and $(\text { ker } K)^{\perp}$ to obtain topological excitations. However, the charge vector after this projection is not necessarily an integer vector, so we need the former condition to ensure that topological quasiparticles/vortices indeed exist.
} 
The appearance of the topological order can be explained also by a 't Hooft anomaly [43, 48-50] (see also [51-69] for recent applications), which is an obstruction in gauging a global symmetry. To see this, we introduce background gauge fields for a pair of discrete 1-form and 2-form symmetries. Suppose that $p \in(\operatorname{coker} K)^{\perp}$ generates $\mathbb{Z}_{N^{(1)}}$ 1-form symmetry and $q \in(\operatorname{ker} K)^{\perp}$ generates $\mathbb{Z}_{N^{(2)}}$ 2-form symmetry. The integers $N^{(1)}$ and $N^{(2)}$ are determined as the minimal integers so that the following $\Lambda^{(1)}$ and $\Lambda^{(2)}$ are integer vectors,

$$
\Lambda_{A}^{(1)}=N^{(1)}\left[K^{+} p\right]_{A}, \quad \Lambda_{i}^{(2)}=N^{(2)}\left[q^{T} K^{+}\right]_{i} .
$$

We will couple the system to background gauge fields corresponding to those symmetries. To do this, it is convenient to write the $B F$ coupling term of the action as

$$
S_{\mathrm{BF}}=\mathrm{i} \frac{K_{i A}}{2 \pi} \int_{M_{5}} \mathrm{~d} b_{i} \wedge \mathrm{d} a_{A},
$$

where $M_{5}$ is a 5-dimensional manifold with $\partial M_{5}=M_{4} . S_{\mathrm{BF}}$ defines the four-dimensional local theory because $S_{\mathrm{BF}} \in 2 \pi \mathrm{i} \mathbb{Z}$ on closed five-dimensional manifolds. We can introduce background gauge fields as

$$
S_{\mathrm{BF}, \text { gauged }}=\mathrm{i} \frac{K_{i A}}{2 \pi} \int_{M_{5}}\left(\mathrm{~d} b_{i}+\Lambda_{i}^{(2)} \mathcal{B}\right) \wedge\left(\mathrm{d} a_{A}+\Lambda_{A}^{(1)} \mathcal{A}\right)
$$

where $\mathcal{A}$ is $\mathbb{Z}_{N^{(1)}}$ 2-form gauge field and $\mathcal{B}$ is a $\mathbb{Z}_{N^{(2)}} 3$-form gauge field, that can be written as

$$
N^{(1)} \mathcal{A}=\mathrm{d} \mathcal{A}^{\prime}, \quad N^{(2)} \mathcal{B}=\mathrm{d} \mathcal{B}^{\prime} .
$$

Since $S_{\mathrm{BF} \text {,gauged }}$ is obtained by the minimal coupling procedure, it is manifestly invariant under the $\mathbb{Z}_{N^{(1)}}$ 1-form and $\mathbb{Z}_{N^{(2)}}$ 2-form gauge transformations. However, $S_{\mathrm{BF} \text {,gauged }}$ is no longer well-defined as a four-dimensional theory unless $\left(q^{T} K^{+} p\right) \in \mathbb{Z}$. Indeed, using $q^{T} K^{+} K=q^{T}$ and $K K^{+} p=p$, we find that

$$
\begin{aligned}
S_{\mathrm{BF}, \text { gauged }}= & 2 \pi \mathrm{i} \int_{M_{5}}\left(\frac{\mathrm{d} b^{T}}{2 \pi} \wedge K \frac{\mathrm{d} a}{2 \pi}+\frac{N^{(2)} \mathcal{B}}{2 \pi} \wedge \frac{q^{T} \mathrm{~d} a}{2 \pi}+\frac{\mathrm{d} b^{T} p}{2 \pi} \wedge \frac{N^{(1)} \mathcal{A}}{2 \pi}\right) \\
& +2 \pi \mathrm{i}\left(q^{T} K^{+} p\right) \int_{M_{5}} \frac{N^{(2)} \mathcal{B}}{2 \pi} \wedge \frac{N^{(1)} \mathcal{A}}{2 \pi} .
\end{aligned}
$$

The first line on the right-hand-side is well-defined as a local functional on $M_{4}=\partial M_{5}$ modulo $2 \pi \mathrm{i}$, but the second line is not if $q^{T} K^{+} p \notin \mathbb{Z}$ and is only well-defined modulo $2 \pi \mathrm{i}\left(q^{T} K^{+} p\right)$. This means that the partition function $Z_{M_{4}}[\mathcal{A}, \mathcal{B}]$ of $(2.27)$ with the background gauge field $\mathcal{A}$ and $\mathcal{B}$ cannot be gauge invariant as a four-dimensional field theory, which indicates a 't Hooft anomaly. To make it anomaly free, we need to regard it as a boundary theory of the five-dimensional symmetry-protected topological states,

$$
Z_{M_{4}}[\mathcal{A}, \mathcal{B}] \exp \left[-2 \pi \mathrm{i}\left(q^{T} K^{+} p\right) \int_{M_{5}} \frac{N^{(2)} \mathcal{B}}{2 \pi} \wedge \frac{N^{(1)} \mathcal{A}}{2 \pi}\right] .
$$

This is an anomaly matching condition by Callan-Harvey's anomaly-inflow mechanism [70]. The existence of a 't Hooft anomaly indicates that the ground state cannot be a trivially gapped state. In the current case, the anomaly matching condition is satisfied by the appearance of topological order. 


\section{Color-flavor locked phase of QCD}

As an application of the general framework discussed so far, let us discuss the color superconductivity in dense QCD [34]. We will see that there exists the nontrivial mutual statistics between test quark and minimal winding vortices [35], and it indicates the emergence of a $\mathbb{Z}_{3} 2$-form symmetry [34]. We show that any vortex operators show algebraic decay instead of the surface law, and thus this 2-form symmetry is unbroken. This observation is important to extend the notion of quark-hadron continuity [28] to the continuity as quantum phases at zero temperature [34].

We note that this section is the follow up of the previous paper [34] by the same authors with more detailed presentations.

\subsection{Generalized $B F$ theory for CFL phase}

A color superconducting phase is realized by the condensation of the Cooper pairs of quarks. Let us consider the 3-color and 3-flavor QCD with flavor-degenerate mass of fundamental quarks, then the most attractive channel between quarks near the Fermi sea is anti-symmetric in both color and flavor. The order parameter field in the effective gauged Ginzburg-Landau description is thus the diquark condensate, $\Phi_{\alpha i}$, where $\alpha$ and $i$ are indices of the anti-fundamental representation for color and flavor, respectively. This complex scalar fields $\Phi$ has charge 2 under U(1) quark number symmetry. In the mean-field approximation with an appropriate gauge, we get $\left\langle\Phi_{\alpha i}\right\rangle \propto \delta_{\alpha i}$ at sufficiently large quark chemical potentials, and the diagonal transformation of color and flavor is unbroken,

$$
\frac{\mathrm{SU}(3)_{\mathrm{c}} \times \mathrm{SU}(3)_{\mathrm{f}} \times \mathrm{U}(1)}{\mathbb{Z}_{3} \times \mathbb{Z}_{3}} \rightarrow \frac{\mathrm{SU}(3)_{\mathrm{c}+\mathrm{f}}}{\mathbb{Z}_{3}} \times \mathbb{Z}_{2} .
$$

This phase is therefore called color-flavor locking (CFL) [27-29], and there is a massless NG boson associated with the spontaneously broken $\mathrm{U}(1)$ symmetry.

Here, let us formally generalize the flavor and color group to be $\mathrm{SU}(N)_{\mathrm{f}}$ and $\mathrm{SU}(N)_{\mathrm{c}} \cdot{ }^{12}$ The scalar field $\Phi$ is taken to be in the anti-fundamental representation both for $\mathrm{SU}(N)_{\mathrm{c}}$ color group and $\mathrm{SU}(N)_{\mathrm{f}}$ flavor group, although it is no longer related to the Cooper pair of fundamental quarks ${ }^{13}$ when $N \neq 3$. The effective Lagrangian of the CFL phase is given by a gauged Ginzburg-Landau model,

$$
S=\frac{1}{2 g_{\mathrm{YM}}^{2}}|G|^{2}+\frac{1}{2}\left|\left(\mathrm{~d}+\mathrm{i} a_{\mathrm{SU}(N)}\right) \Phi\right|^{2}+V_{\mathrm{eff}}\left(\Phi^{\dagger} \Phi,|\operatorname{det}(\Phi)|\right),
$$

where $a_{\mathrm{SU}(N)}$ is the $\mathrm{SU}(N)_{\mathrm{c}}$ color gauge field, $G$ is its field strength, and the effective potential $V_{\text {eff }}$ depends only on the color-singlet order parameters, $\Phi^{\dagger} \Phi$ and $\operatorname{det}(\Phi)$. We here choose $V_{\text {eff }}$ so that it has the minima at

$$
\Phi^{\dagger} \Phi=\Delta^{2} \mathbf{1}
$$

\footnotetext{
${ }^{12}$ A similar gauged GL model with $\mathrm{U}(N)$ c gauge group is considered in ref. [71]. In this case, all the gauge fields are massive and there is no massless NG modes.

${ }^{13}$ Only when $N=3$, the two-index anti-symmetric representation is the same with the anti-fundamental representation.
} 
The mean-field approximation then realizes the symmetry breaking pattern of the CFL phase,

$$
\frac{\mathrm{SU}(N)_{\mathrm{c}} \times \mathrm{SU}(N)_{\mathrm{f}} \times \mathrm{U}(1)}{\mathbb{Z}_{N} \times \mathbb{Z}_{N}} \rightarrow \frac{\mathrm{SU}(N)_{\mathrm{c}+\mathrm{f}}}{\mathbb{Z}_{N}} \times \mathbb{Z}_{2} .
$$

Here, we again assign the charge 2 to $\Phi$ under U(1) symmetry as in the case of $N=3$ QCD, although this is not mandatory for $N \neq 3$ because of the absence of its interpretation as Cooper pairs.

In order to apply the formulation in section 2, we take a gauge fixing so that the diquark field $\Phi$ satisfying (4.3) is taken to be diagonal,

$$
\Phi=\Delta \operatorname{diag}\left(\mathrm{e}^{\phi_{1}}, \cdots, \mathrm{e}^{\phi_{N}}\right),
$$

where $\phi_{i}$ are $2 \pi$-periodic compact scalar fields to denote the phase fluctuations. With this gauge fixing, only Abelian part of the gauge fields remain. As an example of the Cartan generators of $\mathrm{SU}(N)$, let us take the Gell-Mann-type matrices,

$\tau_{1}=\operatorname{diag}(1,-1,0, \ldots, 0), \tau_{2}=\operatorname{diag}(1,1,-2,0, \ldots, 0), \ldots, \tau_{N-1}=\operatorname{diag}(1, \ldots, 1,-(N-1))$.

Since we can easily find that

$$
\exp \left(\frac{2 \pi \mathrm{i}}{n+1} \tau_{n}\right)=\exp \left(\frac{2 \pi \mathrm{i}}{n+1} \tau_{n+1}\right)
$$

for $n=1, \ldots, N-2, \mathbb{Z}_{n+1} \subset \mathrm{U}(1)_{\tau_{n}} \times \mathrm{U}(1)_{\tau_{n+1}}$ does not generate gauge transformations, so the gauge redundancy is represented by

$$
\frac{\mathrm{U}(1)_{\tau_{1}} \times \mathrm{U}(1)_{\tau_{2}} \cdots \times \mathrm{U}(1)_{\tau_{N-1}}}{\mathbb{Z}_{2} \times \cdots \times \mathbb{Z}_{N-1}} \subset \mathrm{SU}(N) .
$$

Let us denote the $\mathrm{U}(1)_{\tau_{A}}$ gauge field as $a_{A}$. The scalar field $\mathrm{e}^{\mathrm{i} \phi_{i}}$ has a charge $\left(\tau_{A}\right)_{i i}$ under $\mathrm{U}(1)_{A}$, and thus the charge matrix $K$ of this theory is given by a $N \times(N-1)$ matrix,

$$
K_{i A}=\left(\begin{array}{cccc}
1 & 1 & \ldots & 1 \\
-1 & 1 & \ldots & 1 \\
0 & -2 & \ldots & 1 \\
\vdots & \vdots & \ddots & \vdots \\
0 & 0 & \ldots & -(N-1)
\end{array}\right)
$$

Taking the Abelian duality, we get the effective theory of the form (2.6),

$$
S=\frac{\Delta^{-2}}{8 \pi^{2}} \int\left|\mathrm{d} b_{i}\right|^{2}+\frac{\operatorname{tr}\left(\tau_{A} \tau_{B}\right)}{2 g_{\mathrm{YM}}^{2}} \int \mathrm{d} a_{A} \wedge \star \mathrm{d} a_{B}+\frac{\mathrm{i}}{2 \pi} \int \mathrm{d} b^{T} \wedge K \mathrm{~d} a .
$$

Although 2-form gauge fields $b_{i}$ follow the canonical normalization, the $\mathrm{U}(1)$ 1-form gauge fields $a_{A}$ obey the non-canonical normalization

$$
Q_{A B} \int \mathrm{d} a_{B} \in 2 \pi \mathbb{Z},
$$

with $Q_{A B}=K_{(i=A), B}$ for $A, B=1, \ldots, N-1$ because the gauge group is (4.8). 
As a result of the diquark condensation, all the gluons become massive by Higgs mechanism and there is no massless 1 -form gauge field. This is equivalent to $\operatorname{dim}(\operatorname{ker} K)=0$, and thus we can drop the kinetic term of the gauge field in (4.8) when discussing the low-energy physics. On the other hand,

$$
\operatorname{coker} K=\mathbb{R}\left(\begin{array}{c}
1 \\
\vdots \\
1
\end{array}\right),
$$

and correspondingly there is one massless NG mode, $\mathrm{d} b_{0} \equiv \mathrm{d}\left(b_{1}+\cdots+b_{N}\right)$, which is associated with the spontaneous breaking of $\mathrm{U}(1)$ baryon number symmetry. Thanks to the permutation invariance of the kinetic term of $b_{i}$, which comes out of $\mathrm{U}(N)$ flavor symmetry, the mixed kinetic term between $b_{0}$ and massive modes does not arise, and (2.26) is satisfied. Therefore, we can obtain the effective theory of the form (2.27),

$$
S_{\text {eff }}[b, a]=\frac{G_{0}^{b}}{2} \int\left|\mathrm{d} b_{0}\right|^{2}+\frac{\mathrm{i}}{2 \pi} \int b^{T} \wedge K \mathrm{~d} a,
$$

with $G_{0}^{b}=\Delta^{2} / 4 \pi^{2} N^{2}$.

Let us perform the basis change in section 2.3.2 with $M=Q^{-1}$ so that we work on the canonically normalized gauge fields $\widetilde{a}_{A}$, i.e. $\int \mathrm{d} \widetilde{a}_{A} \in 2 \pi \mathbb{Z}$ :

$$
\widetilde{a}_{A}=Q_{A B} a_{B} .
$$

The effective action (4.13) becomes

$$
S_{\text {eff }}[b, \widetilde{a}]=\frac{G_{0}^{b}}{2} \int\left|\mathrm{d} b_{0}\right|^{2}+\frac{\mathrm{i}}{2 \pi} \int b^{T} \wedge \widetilde{K} \mathrm{~d} \widetilde{a},
$$

with the new charge matrix $\widetilde{K}$,

$$
\widetilde{K}=K Q^{-1}=\left(\begin{array}{cccc}
1 & 0 & \ldots & 0 \\
0 & 1 & \ldots & 0 \\
\vdots & \vdots & \ddots & \vdots \\
0 & 0 & \ldots & 1 \\
-1 & -1 & \ldots & -1
\end{array}\right)
$$

Note that this transformation does not violate the condition (2.26) because it only changes the massive gauge fields $a_{A}$. We can directly obtain the effective action (4.15) if we use the another Cartan generator,

$$
\widetilde{\tau}_{i}=\operatorname{diag}(0, \ldots, 0, \overbrace{1}^{i \text {-th }}, 0, \ldots, 0,-1),
$$

with $i=1, \ldots, N-1$, instead of (4.6). With this Cartan basis, the Abelian subgroup of $\mathrm{SU}(N)$ takes the simper form as

$$
\mathrm{U}(1)_{\widetilde{\tau}_{1}} \times \cdots \times \mathrm{U}(1)_{\widetilde{\tau}_{N-1}} \subset \mathrm{SU}(N),
$$

compared with (4.8). 
We can apply the extra transformation to obtain the Smith normal form,

$$
\widetilde{K}=U^{-1} \widetilde{K}^{\prime}
$$

with

$$
U^{-1}=\left(\begin{array}{ccccc}
1 & 0 & \ldots & 0 & 0 \\
0 & 1 & \ldots & 0 & 0 \\
\vdots & \vdots & \ddots & \vdots & \vdots \\
0 & 0 & \ldots & 1 & 0 \\
-1 & -1 & \ldots & -1 & 1
\end{array}\right), \quad \widetilde{K}^{\prime}=\left(\begin{array}{cccc}
1 & 0 & \ldots & 0 \\
0 & 1 & \ldots & 0 \\
\vdots & \vdots & \ddots & \vdots \\
0 & 0 & \ldots & 1 \\
0 & 0 & \ldots & 0
\end{array}\right)
$$

The transformation violates (2.26), and the result in section 3 cannot be naively applied due to the mixed kinetic term between the massless and heavy degrees of freedom. We therefore do not take the Smith normal form in this section, but put the detailed computation with the Smith normal form in appendix D.

\subsection{Emergent higher-form symmetry of CFL phase}

Since the Higgs mechanism makes all the gauge fields massive, the Wilson loops

$$
W_{\widetilde{A}}(C)=\exp \left(\mathrm{i} \int_{C} \widetilde{a}_{A}\right)
$$

obey the perimeter law. They therefore generate discrete 2-form transformations on the vortex operators, ${ }^{14}$

$$
V_{i}(S)=\exp \left(\mathrm{i} \int_{S} b_{i}\right) \mapsto \mathrm{e}^{2 \pi \mathrm{i} \widetilde{K}_{A i}^{+}} V_{i}(S) .
$$

The Moore-Penrose inverse of $\widetilde{K}$ is given by

$$
\widetilde{K}_{A i}^{+}=\left[Q K^{+}\right]_{A i}=\delta_{A i}-\frac{1}{N}
$$

and thus this is the $\mathbb{Z}_{N}$ 2-form symmetry [34]. This shows that the test quark and the minimal winding vortex has the $\mathbb{Z}_{N}$ mutual statistics [35]:

$$
\left\langle W_{\widetilde{A}}(C) V_{i}(S)\right\rangle=\exp \left(-\frac{2 \pi \mathrm{i}}{N} \operatorname{Lk}(C, S)\right)\left\langle V_{i}(S)\right\rangle .
$$

Note that this phase rotation is a subgroup of the U(1) 2-form symmetry.

Let us show that the above $\mathbb{Z}_{N} 2$-form symmetry is unbroken [34]. To show it, we have to see that any vortex operators $V_{p}(S)$ of charge $p$ decay faster than the perimeter (surface) law when the charge $p$ is nontrivial under $\mathbb{Z}_{N}$ 2-form symmetry. $V_{p}$ is topological if and only if $p \in(\operatorname{coker} \widetilde{K})^{\perp}$, and those operators are generated by

$$
V_{i}(S) V_{i+1}(S)^{-1} \text {. }
$$

Since these operators are neutral under $\mathbb{Z}_{N}$, we find that this symmetry is unbroken. This also means that the theory has no 1-form symmetry since any topological surface operators are neutral with the Wilson loops. As a consequence, the CFL phase at the zero temperature acquires the emergent $\mathbb{Z}_{N} 2$-form symmetry, but there is no topological order since it is unbroken.

\footnotetext{
${ }^{14}$ The vortices with minimal energy in the CFL are called non-Abelian vortices or semi-superfluid vortices [30]. See ref. [32] for a review.
} 


\subsection{Implications for the quark-hadron continuity scenario}

Let us comment on the physical consequences of the unbroken $\mathbb{Z}_{3} 2$-form symmetry regarding the quark-hadron continuity scenario $[28,34]$. Conventionally, the phases of matter have been classified by the (0-form) symmetry of the system. If there are two phases with the same symmetry, they are considered to be in the same phase. It means that there exists a certain deformation of the Hamiltonian by which the two phases can be continuously connected. When we consider quantum phases of matter, there can be phases with the same 0-form symmetry but are distinguished by different topological orders. Here, we have shown here that the CFL phase does not have a deconfined discrete gauge field and that implies the absence of topological order, although the appearance of fractional braiding phase has a certain similarity to the nature of a topological ordered state. The braiding phase is shown to be a direct consequence of a (unbroken) $\mathbb{Z}_{3} 2$-form symmetry. In addition, the system does not have a 1-form symmetry, and hence there is not mixed 't Hooft anomaly of discrete higher-form symmetries, which allows for a topologically trivial ground state. So, as far as the ground state property is concerned, the CFL can be continuously connected to a nucleon superfluid phase, which presumably has a trivial topological structure because of the absence of deconfined gluons. Thus, we have extended the continuity scenario to zero temperature.

Note that this does not necessarily mean that there is no phase transition between nucleon superfluidity and the CFL phase as a function of baryon chemical potential $\mu_{\mathrm{B}}$. Even if two phases have the same symmetry, as liquid and vapor phases of water, there can be a phase transition, depending on which path one would take in the parameter space. The same is true for the quark-hadron continuity at finite temperatures [28]. In order to predict what would happen along a particular path in a parameter space like $\left(T, \mu_{\mathrm{B}}\right)$, a more detailed approach is necessary.

One might argue that the existence of the (unbroken) $\mathbb{Z}_{3} 2$-form symmetry gives us a distinction of the CFL and nucleon superfluidity. However, as we have shown, the discrete $\mathbb{Z}_{3}$ symmetry is in fact a subgroup of the U(1) 2-form symmetry, and this symmetry is associated with the existence of a massless U(1) NG mode. Because this mode also exists in a nuclear superfluid phase, the continuous 2-form symmetry is also present in this phase. Therefore, nucleon superfluidity and the CFL phase have the same 0-form and higher-form symmetries.

\section{Example of superfluidity with topological order}

In this section, let us discuss an example of superfluidity with topological order. We consider the generalized $B F$ theory with the action (4.13),

$$
S_{\text {eff }}[b, a]=\frac{G_{0}^{b}}{2} \int\left|\mathrm{d} b_{0}\right|^{2}+\frac{\mathrm{i}}{2 \pi} \int b^{T} \wedge K \mathrm{~d} a,
$$

with the $K$ matrix (4.9). In the case of the CFL phase, the U(1) 1-form gauge fields $a_{A}$ obey the non-canonical normalization, (4.11), and no topological order appears. In this section, we instead assume the canonical normalization of gauge fields, $\int \mathrm{d} a_{A} \in 2 \pi \mathbb{Z}$. 
All the Wilson loops $W_{A}(C)=\exp \left(\mathrm{i} \int_{C} a_{A}\right)$ are topological because the theory (5.1) is in the Higgs phase. The gauge-invariant correlation functions are obtained by

$$
\left\langle W_{A}(C) V_{i}(S)\right\rangle=\exp \left(-2 \pi \mathrm{i} K_{A i}^{+} \operatorname{Lk}(C, S)\right)\left\langle V_{i}(S)\right\rangle .
$$

The Moore-Penrose inverse of (4.9) is given by

$$
K^{+}=\left(\begin{array}{cccccc}
\left(1-\frac{1}{2}\right) & -\frac{1}{2} & 0 & 0 & \ldots & 0 \\
\left(\frac{1}{2}-\frac{1}{3}\right) & \left(\frac{1}{2}-\frac{1}{3}\right) & -\frac{1}{3} & 0 & \ldots & 0 \\
\left(\frac{1}{3}-\frac{1}{4}\right) & \left(\frac{1}{3}-\frac{1}{4}\right) & \left(\frac{1}{3}-\frac{1}{4}\right) & -\frac{1}{4} & \ldots & 0 \\
\vdots & \vdots & \vdots & \vdots & \ddots & \vdots \\
\frac{1}{N(N-1)} & \frac{1}{N(N-1)} & \frac{1}{N(N-1)} & \frac{1}{N(N-1)} & \ldots & -\frac{1}{N}
\end{array}\right),
$$

and we can now find the particle-vortex statistics explicitly.

The set of topological Wilson lines generates $\mathbb{Z}_{2} \times \cdots \mathbb{Z}_{N-1} \times \mathbb{Z}_{N} 2$-form symmetry. To see this, let us take the basis of charge vectors $q_{n}$ of Wilson lines as

$$
q_{1}=\left(\begin{array}{c}
1 \\
0 \\
0 \\
\vdots \\
0
\end{array}\right), q_{2}=\left(\begin{array}{c}
1 \\
1 \\
0 \\
\vdots \\
0
\end{array}\right), \ldots, q_{N-1}=\left(\begin{array}{c}
1 \\
1 \\
1 \\
\vdots \\
1
\end{array}\right),
$$

that is, $W_{q_{n}}(C)=\exp \left(\mathrm{i} \int_{C}\left(a_{1}+\cdots+a_{n}\right)\right)$. The fractional phase is determined as

$$
q_{n}^{T} K^{+}=\overbrace{\left(-\frac{1}{n+1}, \ldots,-\frac{1}{n+1}\right.}^{n+1}, \overbrace{0, \ldots, 0}^{N-1-n}) \bmod 1 .
$$

Therefore, $W_{q_{n}}$ generates $\mathbb{Z}_{n+1}$ 2-form symmetry.

Similarly, we can find the $\mathbb{Z}_{2} \times \cdots \times \mathbb{Z}_{N-1} 1$-form symmetry. Topological surface operators are given by the vortex charges $p \in(\operatorname{coker} K)^{\perp}$, and its basis can be chosen as

$$
p_{1}=\left(\begin{array}{c}
1 \\
-1 \\
0 \\
\vdots \\
0 \\
0
\end{array}\right), p_{2}=\left(\begin{array}{c}
0 \\
1 \\
-1 \\
\vdots \\
0 \\
0
\end{array}\right), \ldots, p_{N-1}=\left(\begin{array}{c}
0 \\
0 \\
0 \\
\vdots \\
1 \\
-1
\end{array}\right) \text {, }
$$

that is, $V_{p_{m}}(S)=\exp \left(\mathrm{i} \int_{S}\left(b_{m}-b_{m+1}\right)\right)$. The topological surface operator $V_{p_{m}}$ generates $\mathbb{Z}_{m} 1$-form symmetry for $m=2, \ldots, N-1$. Indeed, this can be found by

$$
q_{n}^{T} K^{+} p_{m}=-\frac{1}{n+1} \delta_{n+1, m} \bmod 1
$$

and thus $W_{q_{n}}$ and $V_{p_{n+1}}$ have the mutual $\mathbb{Z}_{n+1}$ statistics for $n=1, \ldots, N-2$. This shows that $\mathbb{Z}_{2} \times \cdots \times \mathbb{Z}_{N-1}$ 1-form and 2-form symmetries are spontaneously broken, and thus this theory supports a topological order. 
The $\mathbb{Z}_{N} 2$-form symmetry generated by $W_{q_{N-1}}$ is unbroken. This fact comes out of the same discussion given in section 4. All the vortex operators charged under $\mathbb{Z}_{N}$ are coupled to NG boson, and thus those vortices are logarithmically confined. In other words, $\mathbb{Z}_{N} 2$-form symmetry is a subgroup of U(1) 2 -form symmetry defining the vortex winding numbers, and thus the generalized Coleman-Mermin-Wagner theorem prohibits its spontaneous breaking.

We therefore conclude that the theory describes superfluidity of one NG mode with $\mathbb{Z}_{2} \times \cdots \times \mathbb{Z}_{N-1}$ topological order.

\section{Summary and outlook}

We have discussed a general effective theory for superfluids with topological order. Starting from a gauged GL model, we have derived a low-energy gauge theory written in terms of 2 -form and 1-form gauge fields. The theory has a structure of a $B F$ theory with a nonsquare $K$ matrix that can have a nontrivial kernel/cokernel, coupled with massless NG modes corresponding to the breaking of U(1) symmetries. Physical spectrum are classified according to the structure of the $K$ matrix. We have discussed the symmetry of the theory. There can be discrete 1-form and 2-form symmetries as well as U(1) 1-form and 2-form symmetries. We have shown that the correlation of vortices and quasiparticles is written in terms of the topological information of vortex surfaces and quasiparticle world-lines. We have discussed how to identify the presence of topological order, which is summarized in the condition ( $)$. If there is a vortex operator whose average obeys perimeter law, the 2 -form symmetry is broken, which indicates the topological order.

As an application of the framework, we have discussed the CFL phase of dense QCD matter. We have analyzed the higher-symmetry of the phase and shown that color Wilson loops and vortices show fractional braiding as a consequence of $\mathbb{Z}_{3} 2$-form symmetry. We have shown that the nuclear superfluid phase and the CFL phase have the same symmetry including higher-form ones, which extends the quark-hadron continuity scenario to zero temperature. We have also discussed an example of superfluidity with topological order in section 5 and we identified the symmetry and topological order in this system.

We believe that the framework discussed in this paper would be useful in identifying the topological structure in systems where topological order and massless modes coexist, which include high $T_{\mathrm{c}}$ cuprate superconductivity.

\section{Acknowledgments}

The work of Y.T. was supported by JSPS Overseas Fellowship. The work of Y.H. was supported in part by the Korean Ministry of Education, Science and Technology, Gyeongsangbuk-do and Pohang City for Independent Junior Research Groups at the Asia Pacific Center for Theoretical Physics. 


\section{A Moore-Penrose inverse}

Here we summarize the properties of the Moore-Penrose inverse that are used in the paper.

Let $K_{i A}$ be a $|i| \times|A|$ matrix with real entries. The $|A| \times|i|$ matrix $\left[K^{+}\right]_{A i}$ that satisfies the following 4 conditions,

$$
\begin{aligned}
K K^{+} K & =K, \\
K^{+} K K^{+} & =K^{+}, \\
\left(K K^{+}\right)^{T} & =K K^{+}, \\
\left(K^{+} K\right)^{T} & =K^{T} K,
\end{aligned}
$$

is called the Moore-Penrose inverse of $K$, which is a generalization of the inverse matrix. For any matrix, its Moore-Penrose inverse always exists and is unique. If linear equations

$$
K x=b,
$$

have any solution, it has to take the form

$$
x=K^{+} b+\left[\mathbf{1}-K^{+} K\right] w,
$$

where $w$ is an arbitrary vector. Solutions exist if and only if $K K^{+} b=b$. The kernel and cokernel of $K$ and $K^{+}$are related as follows,

$$
\begin{aligned}
& \operatorname{ker} K^{+}=\operatorname{coker} K, \\
& \text { coker } K^{+}=\operatorname{ker} K .
\end{aligned}
$$

Using $K^{+}$and $K$, we can define orthogonal projection matrices in the following way:

$$
\begin{aligned}
P & \equiv 1-K^{+} K, \quad \text { projection to ker } K, \\
P^{c} & \equiv 1-K K^{+}, \quad \text { projection to coker } K .
\end{aligned}
$$

Given two matrices $K$ and $L,(K L)^{+}=L^{+} K^{+}$is true if and only if either of the following conditions is satisfied:

$$
\begin{aligned}
K^{T} K & =1, \\
L L^{T} & =1, \\
L & =K^{T}, \\
\operatorname{dim}(\operatorname{ker} K) & =\operatorname{dim}(\operatorname{coker} L)=0 .
\end{aligned}
$$

\section{B Delta function forms}

For $n$-dimensional submanifold $M_{n}$ on $D$-dimensional manifold, a $D-n$ dimensional delta function form supported on $M_{n}$ is defined by

$$
\int_{M_{n}} A=\int A \wedge \delta^{\perp}\left(M_{n}\right)
$$

where $A$ is any $n$-form. 
An exterior derivative of a delta function form is given by

$$
\mathrm{d} \delta^{\perp}\left(M_{n}\right)=(-)^{D-n+1} \delta^{\perp}\left(\partial M_{n}\right),
$$

where $\partial M_{n}$ is the boundary of $M_{n}$. The delta function form is odd under flipping of the orientation of $M_{n}$,

$$
\delta^{\perp}\left(-M_{n}\right)=-\delta^{\perp}\left(M_{n}\right) .
$$

Let $M_{n}$ and $M_{m}$ be $n$ - and $m$-dimensional submanifold of the total space. The intersection of those two manifolds, $M_{n} \cap M_{m}$, is $D-n-m$-dimensional manifold. The delta function form of $M_{n} \cap M_{m}$ is given by

$$
\delta^{\perp}\left(M_{n} \cap M_{m}\right)=\delta^{\perp}\left(M_{n}\right) \wedge \delta^{\perp}\left(M_{m}\right) .
$$

When $n+m=D$, the interaction regions are points. The number of intersection is counted by

$$
\mathrm{I}\left(M_{n}, M_{m}\right)=\int \delta^{\perp}\left(M_{n}\right) \wedge \delta^{\perp}\left(M_{m}\right),
$$

which is an integer.

\section{Derivation of the braiding phase (3.13)}

Here let us derive eq. (3.13). We would like to compute the path integral,

$$
\frac{1}{Z} \int \mathcal{D} a \mathcal{D} b \exp \left(-S_{\text {eff }}+\mathrm{i} \int(R p)_{i} b_{i} \wedge j_{v}+\mathrm{i} \int\left(q^{T} Q\right)_{A} a_{A} \wedge j_{p}\right)
$$

where $S_{\text {eff }}$ is given in eq. (2.27), $j_{p}$ is a quasiparticle current, and $j_{v}$ is a vortex current. The sources are represented by

$$
j_{v}=\delta^{\perp}(S), \quad j_{p}=\delta^{\perp}(C),
$$

where $C$ and $S$ are world-line and world-sheet of the particle and vortex, and $\delta^{\perp}(S)\left(\delta^{\perp}(C)\right)$ are the 2-form (1-form) valued delta function whose support is $S(C)$.

By integrating out the fields, the path integral localized around the solutions of classical equations of motions. Note that the kinetic terms of $a_{0}$ and $b_{0}$ can expressed as

$$
\begin{aligned}
\frac{1}{2} \int G_{A B}^{a} \mathrm{~d}\left(a_{0}\right)_{A} \wedge \star \mathrm{d}\left(a_{0}\right)_{A} & =\frac{1}{2} \int\left[P^{a} G^{a} P^{a}\right]_{A B} \mathrm{~d} a_{A} \wedge \star \mathrm{d} a_{B}, \\
\frac{1}{2} \int G_{i j}^{b} \mathrm{~d}\left(b_{0}\right)_{i} \wedge \star \mathrm{d}\left(b_{0}\right)_{i} & =\frac{1}{2} \int\left[P^{b} G^{b} P^{b}\right]_{i j} \mathrm{~d} b_{i} \wedge \star \mathrm{d} b_{j} .
\end{aligned}
$$

Integrating out $b$, we obtain

$$
\frac{1}{2 \pi} K_{i A} \mathrm{~d} a_{A}+(R p)_{i} j_{v}-\left[P^{b} G^{b} P^{b}\right]_{i j} \mathrm{~d} \star \mathrm{d} b_{j}=0 .
$$

Multiplying $K^{T}$ from left,

$$
\frac{1}{2 \pi}\left[K^{T} K\right]_{B A} \mathrm{~d} a_{A}+\left[K^{T} R p\right]_{B} j_{v}=0,
$$


where we used a property of Moore-Penrose inverse, $K^{T} P^{b}=K^{T}-K^{T} K K^{+}=0$. We can solve this for $a_{A}$ as

$$
a_{A}=-2 \pi\left[K^{+} R p\right]_{A} \mathrm{~d}^{-1} j_{v}+\left(a_{0}\right)_{A},
$$

up to a closed form. Here, we used $\left[K^{T} K\right]^{+} K^{T}=K^{+}$, and the contribution from massless components $\left(a_{0}\right)_{A}$ is to be determined by using the EOM for $\left(a_{0}\right)_{A} \cdot\left[K^{+} R p\right]_{A}$ is in general a fractional number, and this represents a fractional statistics of vortices and quasiparticles. Likewise, integration of $a_{A}$ leads to

$$
\frac{1}{2 \pi} \mathrm{d} b_{i} K_{i A}+\left(q^{T} Q\right)_{A} j_{p}-\left[P^{a} G^{a} P^{a}\right]_{A B} \mathrm{~d} \star \mathrm{d} a_{B}=0,
$$

which can be solved for $b_{i}$ as

$$
b_{i}=-2 \pi\left[q^{T} Q K^{+}\right]_{i} \mathrm{~d}^{-1} j_{p}+\left(b_{0}\right)_{i} .
$$

Using eqs. (C.9) and (C.7), we can evaluate the following terms as

$$
\begin{aligned}
& S_{\mathrm{BF}}+(R p)_{i} \int b_{i} \wedge j_{v}+\left(q^{T} Q\right)_{A} \int a_{A} \wedge j_{p} \\
& =-2 \pi\left(q^{T} Q K^{+} R p\right) \int \mathrm{d}^{-1} j_{v} \wedge j_{p}+(R p)_{i}\left(b_{0}\right)_{i} \wedge j_{v}+\left(q^{T} Q\right)_{A}\left(a_{0}\right)_{A} \wedge j_{p} \\
& =-2 \pi\left(q^{T} Q K^{+} R p\right) \operatorname{Lk}(S, C)+(R p)_{i}\left(b_{0}\right)_{i} \wedge j_{v}+\left(q^{T} Q\right)_{A}\left(a_{0}\right)_{A} \wedge j_{p}
\end{aligned}
$$

Here, we used

$$
\mathrm{d}^{-1} \delta^{\perp}(C)=-\delta^{\perp}(D), \quad \mathrm{d}^{-1} \delta^{\perp}(S)=\delta^{\perp}(V),
$$

where $D$ and $V$ are chosen so that $C$ and $S$ are their boundaries $(C=\partial D$ and $S=\partial V)$, and

$$
\int \delta^{\perp}(V) \wedge \delta^{\perp}(C)=\mathrm{I}(V, C)=\operatorname{Lk}(S, C)
$$

where $\mathrm{I}(V, C)$ is the intersection number of $V$ and $C . \mathrm{I}(V, C)$ is computed by counting the number of intersection with signs that corresponds to orientation.

By taking a ratio as in eq. (3.13) and using eq. (C.10), we can see that the nontopological contributions related to $\left(b_{0}\right)_{i}$ and $\left(a_{0}\right)_{A}$ cancels out, resulting in eq. (3.13). Even though Wilson loops and vortex operators do not show the perimeter law, the geometry a Wilson loop does not affect a vortex and vice versa, because $\left(a_{0}\right)_{A}$ do not interact with $\left(b_{0}\right)_{i}$. That is why the ratio (3.13) is only determined by the topology of vortices and particle loops.

\section{Notes regarding basis changes}

In this appendix, let us discuss the influence of basis changes of the gauge fields. In particular, when we use a basis that does not satisfy the condition (2.26), the kinetic terms mix the massless and massive modes. Although a basis change may simplify the structure of $B F$ terms, one should properly take into account the effect of the coupling to evaluate correlation functions correctly. We first illustrate this point in a simple example, and then discuss more general cases. 


\section{D.1 Example}

We work on the theory given in section 4 . For simplicity, we mainly focus on the case $N=3$. The $K$ matrix is given by

$$
K_{i A}=\left(\begin{array}{cc}
1 & 0 \\
-1 & 1 \\
0 & -1
\end{array}\right)
$$

One can use the Smith normal form to make the BF terms simpler. The Smith decomposition of this matrix is given by

$$
K_{i B}=U_{i j}^{-1} K_{j A}^{\prime} V_{A B}^{-1}
$$

where

$$
U=\left(\begin{array}{lll}
1 & 0 & 0 \\
1 & 1 & 0 \\
1 & 1 & 1
\end{array}\right), \quad K^{\prime}=\left(\begin{array}{ll}
1 & 0 \\
0 & 1 \\
0 & 0
\end{array}\right), \quad V=\mathbf{1}_{2}
$$

and

$$
U^{-1}=\left(\begin{array}{ccc}
1 & 0 & 0 \\
-1 & 1 & 0 \\
0 & -1 & 1
\end{array}\right)
$$

Let us introduce a new basis for 2-form fields by

$$
c_{i}=b_{j} U_{j i}^{-1} .
$$

In this basis, $B F$ terms are diagonalized as

$$
S_{\mathrm{BF}}=\frac{\mathrm{i}}{2 \pi} \int\left[c_{1} \wedge \mathrm{d} a_{1}+c_{2} \wedge \mathrm{d} a_{2}\right] .
$$

The kinetic term is written as

$$
S_{0}=\frac{1}{2} G_{i j} \int \mathrm{d} b_{i} \wedge \star \mathrm{d} b_{j}=\frac{1}{2}\left[U G U^{T}\right]_{i j} \int \mathrm{d} c_{i} \wedge \star \mathrm{d} c_{j} \equiv \frac{1}{2} G_{i j}^{\prime} \int \mathrm{d} c_{i} \wedge \star \mathrm{d} c_{j} .
$$

In the example of the CFL phase, $G_{i j} \propto \delta_{i j}$ and $G_{i j}^{\prime} \propto\left[U U^{T}\right]_{i j}$, where

$$
U U^{T}=\left(\begin{array}{lll}
1 & 1 & 1 \\
1 & 2 & 2 \\
1 & 2 & 3
\end{array}\right)
$$

Since $U U^{T} \neq \mathbf{1}$, the orthogonality condition (2.26) is violated in the new basis. The action of $\mathbb{Z}_{3}$ symmetry on the rotated basis is

$$
c_{i} \mapsto c_{i}+q_{A} K_{A j}^{+} U_{j i}^{-1} \lambda
$$

where

$$
K_{A j}^{+} U_{j i}^{-1}=\left(\begin{array}{lll}
1 & 0 & -\frac{1}{3} \\
0 & 1 & -\frac{2}{3}
\end{array}\right)
$$

Thus, this symmetry only acts on $c_{3}$. Below, we compare the computation of $\mathbb{Z}_{3} 2$-form symmetry before and after taking the Smith normal form. 


\section{D.2 $\mathbb{Z}_{3}$ symmetry action in the original basis}

We evaluate the following correlation function,

$$
\left\langle\mathrm{e}^{\mathrm{i} q_{2} \int_{C} a_{2}} \mathrm{e}^{\mathrm{i} p_{3} \int_{S} b_{3}}\right\rangle .
$$

First, let us use the original basis. We consider

$$
\begin{aligned}
S\left[b_{1}, b_{2}, b_{3}, a_{1}, a_{2}\right]= & -\frac{g^{2}}{2}\left(\left|\mathrm{~d} b_{1}\right|^{2}+\left|\mathrm{d} b_{2}\right|^{2}+\left|\mathrm{d} b_{3}\right|^{2}\right) \\
& -\frac{\mathrm{i}}{2 \pi}\left[\left(b_{1}-b_{2}\right) \mathrm{d} a_{1}+\left(b_{2}-b_{3}\right) \mathrm{d} a_{2}\right]+\mathrm{i} \int p_{3} b_{3} \wedge \delta^{\perp}(S)+\mathrm{i} \int q_{2} a_{2} \wedge \delta^{\perp}(C) .
\end{aligned}
$$

The correlation function is calculating by the following quantity

$$
Z\left[\delta^{\perp}(C), \delta^{\perp}(S)\right]=\int \prod_{i} \mathcal{D} b_{i} \prod_{A} \mathcal{D} a_{A} \exp \left[S\left[b_{1}, b_{2}, b_{3}, a_{1}, a_{2}\right]\right] .
$$

The integration over $a_{1}$ gives a delta function enforcing $\mathrm{d} b_{1}=\mathrm{d} b_{2}$, and also integrating over $b_{1}$, the action is reduced to

$S\left[b_{2}, b_{3}, a_{2}\right]=-\frac{g^{2}}{2}\left(2\left|\mathrm{~d} b_{2}\right|^{2}+\left|\mathrm{d} b_{3}\right|^{2}\right)-\frac{\mathrm{i}}{2 \pi} \int\left(b_{2}-b_{3}\right) \mathrm{d} a_{2}+\mathrm{i} \int p_{3} b_{3} \wedge \delta^{\perp}(S)+\mathrm{i} \int q_{2} a_{2} \wedge \delta^{\perp}(C)$.

Integration of $a_{2}$ enforces

$$
\mathrm{d} b_{2}=\mathrm{d} b_{3}+2 \pi q_{2} \delta^{\perp}(C),
$$

and integrating over $b_{2}$, the effective action now becomes

$$
S\left[b_{3}\right]=-\frac{g^{2}}{2}\left(2\left|\mathrm{~d} b_{3}+2 \pi q_{2} \delta^{\perp}(C)\right|^{2}+\left|\mathrm{d} b_{3}\right|^{2}\right)+\mathrm{i} \int p_{3} b_{3} \wedge \delta^{\perp}(S) .
$$

By performing $b_{3}$ integration, ${ }^{15}$

$$
Z\left[\delta^{\perp}(S), \delta^{\perp}(C)\right]=C \exp \left[\frac{1}{6 g^{2}}\left(p_{3}\right)^{2}\left(\delta^{\perp}(S),(\delta d)^{-1} \star \delta^{\perp}(S)\right)+2 \pi \mathrm{i} \frac{2}{3} p_{3} q_{2} \operatorname{Lk}(C, S)\right],
$$

where $(x, y) \equiv \int x \wedge \star y$. Therefore, we have

$$
\left\langle W_{q_{2}}(C) V_{p_{3}}(S)\right\rangle=\mathrm{e}^{2 \pi \mathrm{i} \frac{2}{3} \operatorname{Lk}(C, S)}\left\langle V_{p_{3}}(S)\right\rangle .
$$

This relation means that the Wilson loop $\mathrm{e}^{q_{2} \int_{C} a_{2}}$ is the generator of $\mathbb{Z}_{3}$ symmetry. The result is unchanged if we had projected out the massive kinetic terms in the first place. In that case, the kinetic term looks like

$$
S_{0}=-\frac{g^{2}}{2} \frac{1}{3}\left|\mathrm{~d}\left(b_{1}+b_{2}+b_{3}\right)\right|^{2} .
$$

\footnotetext{
${ }^{15}$ Here and hereafter, we neglect the contributions proportional to $\int \delta^{\perp}(C) \wedge \star \delta^{\perp}(C) \propto$ perimeter $(C)$, which can be canceled by local counter terms.
} 


\section{D.3 Calculation in the rotated basis}

Let us evaluate the same correlation function (D.11) in a rotated basis that diagonalizes the $B F$ terms. Since $c_{3}=b_{3}$, the effective action with sources is written as

$$
\begin{aligned}
S\left[c_{1}, c_{2}, c_{3}, a_{1}, a_{2}\right]= & -\frac{g^{2}}{2}\left(\left|\mathrm{~d}\left(c_{1}+c_{2}+c_{3}\right)\right|^{2}+\left|\mathrm{d}\left(c_{2}+c_{3}\right)\right|^{2}+\left|\mathrm{d} c_{3}\right|^{2}\right) \\
& -\frac{\mathrm{i}}{2 \pi}\left[c_{1} \mathrm{~d} a_{1}+c_{2} \mathrm{~d} a_{2}\right]+\mathrm{i} p_{3} c_{3} \wedge \delta^{\perp}(S)+\mathrm{i} q_{2} a_{2} \wedge \delta^{\perp}(C) .
\end{aligned}
$$

Note that, in the rotated basis, there are mixed kinetic terms of massive and massless 2 -form fields. The field $c_{3}$ and $a_{2}$ do not couple in the $B F$ term, so naively the Wilson loop of $a_{2}$ does not seem to generate a phase rotation of the vortex operator of $c_{3}$. In order to reproduce the correct results, the coupling of the massive and massless 2-form fields is important. Integration over $a_{1}$ gives $\mathrm{d} c_{1}=0$, and performing $c_{1}$ integration,

$$
S\left[c_{2}, c_{3}, a_{2}\right]=-\frac{g^{2}}{2}\left(2\left|\mathrm{~d}\left(c_{2}+c_{3}\right)\right|^{2}+\left|\mathrm{d} c_{3}\right|^{2}\right)-\frac{\mathrm{i}}{2 \pi} c_{2} \mathrm{~d} a_{2}+\mathrm{i} p_{3} c_{3} \wedge \delta^{\perp}(S)+\mathrm{i} q_{2} a_{2} \wedge \delta^{\perp}(C) .
$$

Integration over $a_{2}$ gives

$$
\frac{\mathrm{i}}{2 \pi} \mathrm{d} c_{2}=\mathrm{i} q_{2} \delta^{\perp}(C)
$$

and after integrating over $c_{2}$, the resulting action is

$$
S\left[c_{3}\right]=-\frac{g^{2}}{2}\left(2\left|\mathrm{~d} c_{3}+2 \pi q_{2} \delta^{\perp}(C)\right|^{2}+\left|\mathrm{d} c_{3}\right|^{2}\right)+\mathrm{i} \int p_{3} c_{3} \wedge \delta^{\perp}(S) .
$$

This is the same as eq. (D.16), so we have reproduced the same correlation function. Note that the coupling of $c_{2}$ and $\delta^{\perp}(C)$ is introduced through the mixed kinetic term of $c_{2}$ and $c_{3}$. If there is no such coupling, the Wilson loop of $a_{2}$ cannot generate $\mathbb{Z}_{3}$ phase.

\section{D.4 More general cases}

Let us consider a more general situation when the matrix $G_{i j}$ specifying the kinetic terms of $b_{i}$ does not satisfy the condition (2.26) and there are couplings between massless and massive modes. We evaluate the correlation function,

$$
\left\langle W_{q}(C) V_{p}(S)\right\rangle
$$

with generic charge vectors $q$ and $p$. Here we assume that photons $a_{A}$ are all massive and the gauge fields obey the canonical normalization condition, namely $Q=1, R=1$. The effective action is written as

$$
S\left[a_{A}, b_{i}\right]=-\frac{1}{2}\left(\mathrm{~d} b_{i}, G_{i j} \mathrm{~d} b_{j}\right)-\frac{\mathrm{i}}{2 \pi} K_{i A}\left(b_{i}, \star^{-1} \mathrm{~d} a_{A}\right)+\mathrm{i} p_{i}\left(b_{i}, \star^{-1} \delta^{\perp}(S)\right)+\mathrm{i} q_{A}\left(a_{A}, \star^{-1} \delta^{\perp}(C)\right) .
$$

Let $P_{i j}$ the projector to coker $K$. We denote a projected index as

$$
b_{\hat{i}} \equiv P_{i j} b_{j}, \quad b_{\bar{i}} \equiv\left(\delta_{i j}-P_{i j}\right) b_{j} .
$$


Thus, $b_{\hat{i}}$ denotes massless modes and $b_{\bar{i}}$ denotes massive modes. Since we drop the kinetic terms of massive modes, $G_{\bar{i} \bar{j}}=0$. The separability condition (2.26) of the massive and massless modes is written as

$$
G_{\widehat{i j}}=G_{\widehat{i j}}
$$

or equivalently

$$
G_{\widehat{i j}}=0 .
$$

Below we consider the case when this condition is not necessarily satisfied.

Now let us compute eq. (D.24). We evaluate

$$
Z\left[\delta^{\perp}(C), \delta^{\perp}(S)\right]=\int \prod_{A} \mathcal{D} a_{A} \prod_{i} \mathcal{D} b_{i} \exp S\left[a_{A}, b_{i}\right],
$$

with the action (D.25). The integration over $a_{A}$ gives

$$
-\frac{\mathrm{i}}{2 \pi} K_{i A} \mathrm{~d} b_{i}+\mathrm{i} q_{A} \delta^{\perp}(C)=0 .
$$

Let us decompose $b_{i}$ into massless and massive parts,

$$
b_{i}=b_{\bar{i}}+b_{\bar{i}} .
$$

From eq. (D.30), the massive part is determined (up to closed form) as

$$
b_{\bar{i}}=-2 \pi q_{A} K_{A i}^{+} \delta^{\perp}\left(S^{\prime}\right)
$$

where $S^{\prime}$ is a 2-dimensional surface such that $\partial S^{\prime}=C$ and we used $\delta^{\perp}(C)=-\mathrm{d} \delta^{\perp}\left(S^{\prime}\right)$. The effective action becomes

$$
S\left[b_{\hat{i}}\right]=-\frac{1}{2}\left(b_{\hat{i}}+b_{\bar{i}}, G_{i j} \delta \mathrm{d}\left(b_{\hat{j}}+b_{\bar{j}}\right)\right)+\mathrm{i}\left(p_{\bar{i}} b_{\bar{i}}, \star^{-1} \delta^{\perp}(S)\right)+\mathrm{i}\left(p_{\bar{i}} b_{\bar{i}}, \star^{-1} \delta^{\perp}(S)\right),
$$

where $b_{\bar{i}}$ is given by eq. (D.32). The EOM varying $b_{\bar{i}}$ is given by

$$
G_{\widehat{i} j} \delta \mathrm{d}\left(b_{\widehat{j}}+b_{\bar{j}}\right)=\mathrm{i} p_{\widehat{i}} \star \delta^{\perp}(S) .
$$

The massless part is solved as

$$
b_{\hat{i}}=\mathrm{i} G_{\widehat{i} \hat{j}}^{-1} p_{\widehat{j}}(\delta \mathrm{d})^{-1} \star \delta^{\perp}(S)-G_{\widetilde{i} j}^{-1} G_{\widehat{j} \bar{k}} b_{\bar{k}} .
$$

The partition function in the presence of particle and vortex sources is now written as

$$
\begin{aligned}
\ln Z\left[\delta^{\perp}(S), \delta^{\perp}(C)\right]= & -\frac{1}{2} p_{\widehat{i}} G_{\widehat{i} \hat{j}}^{-1} p_{\widehat{j}}\left(\star \delta^{\perp}(S),(\delta \mathrm{d})^{-1} \star \delta^{\perp}(S)\right) \\
& +2 \pi \mathrm{i} q_{A} K_{A \bar{i}}^{+}\left(p_{\bar{i}}-G_{\widehat{i} \hat{j}} G_{\widehat{j} \hat{k}}^{-1} p_{\widehat{k}}\right)\left(\star \delta^{\perp}(S), \delta^{\perp}\left(S^{\prime}\right)\right) .
\end{aligned}
$$

Noting that

$$
\left(\star \delta^{\perp}(S), \delta^{\perp}\left(S^{\prime}\right)\right)=\delta^{\perp}\left(S^{\prime}\right) \wedge \delta^{\perp}(S)=\operatorname{Lk}(C, S)=-\operatorname{Lk}(S, C),
$$

the correlation function is written as

$$
\left\langle W_{q}(C) V_{p}(S)\right\rangle=\left\langle V_{p}(S)\right\rangle \exp \left[-2 \pi \mathrm{i} q_{A} K_{A \bar{i}}^{+}\left(p_{\bar{i}}-G_{\widehat{i} \hat{j}} G_{\widehat{j} \hat{k}}^{-1} p_{\widehat{k}}\right) \operatorname{Lk}(S, C)\right] .
$$

Because of the coupling of massless and massive sector, $G_{\widehat{i} j} \neq 0$ and there are additional contributions to the term proportional to the linking number. Although there is no coupling between $a_{A}$ and $b_{\hat{i}}$ in the $B F$ terms, the Wilson loop can induce a phase rotation of a vortex operator which has components $b_{\hat{i}}$. 
Open Access. This article is distributed under the terms of the Creative Commons Attribution License (CC-BY 4.0), which permits any use, distribution and reproduction in any medium, provided the original author(s) and source are credited.

\section{References}

[1] L.D. Landau, On the theory of phase transitions. I., Zh. Eksp. Teor. Fiz. 11 (1937) 19 [INSPIRE].

[2] V.L. Ginzburg and L.D. Landau, On the theory of superconductivity, Zh. Eksp. Teor. Fiz 20 (1950) 1064 [INSPIRE].

[3] Y. Nambu, Quasiparticles and Gauge Invariance in the Theory of Superconductivity, Phys. Rev. 117 (1960) 648 [INSPIRE].

[4] Y. Nambu and G. Jona-Lasinio, Dynamical model of elementary particles based on an analogy with superconductivity. II, Phys. Rev. 124 (1961) 246 [INSPIRE].

[5] X.G. Wen, Topological Order in Rigid States, Int. J. Mod. Phys. B 4 (1990) 239 [InSPIRE].

[6] X.G. Wen and A. Zee, Topological structures, universality classes, and statistics screening in the anyon superfluid, Phys. Rev. B 44 (1991) 274.

[7] X.-G. Wen, Quantum Field Theory of Many-body Systems: From the Origin of Sound to an Origin of Light and Electrons, Oxford Graduate Texts, Oxford University Press (2007).

[8] T.H. Hansson, V. Oganesyan and S.L. Sondhi, Superconductors are topologically ordered, Annals Phys. 313 (2004) 497 [inSPIRE].

[9] S. Sachdev, Quantum phase transitions, Cambridge University Press (2011).

[10] X. Chen, Z.C. Gu and X.G. Wen, Local unitary transformation, long-range quantum entanglement, wave function renormalization and topological order, Phys. Rev. B 82 (2010) 155138 [arXiv: 1004.3835] [INSPIRE].

[11] M. Levin and X.-G. Wen, Detecting Topological Order in a Ground State Wave Function, Phys. Rev. Lett. 96 (2006) 110405 [cond-mat/0510613] [INSPIRE].

[12] A. Kitaev and J. Preskill, Topological entanglement entropy, Phys. Rev. Lett. 96 (2006) 110404 [hep-th/0510092] [INSPIRE].

[13] R. Dijkgraaf and E. Witten, Mean Field Theory, Topological Field Theory and Multimatrix Models, Nucl. Phys. B 342 (1990) 486 [INSPIRE].

[14] X.G. Wen and A. Zee, A Classification of Abelian quantum Hall states and matrix formulation of topological fluids, Phys. Rev. B 46 (1992) 2290 [InSPIRE].

[15] D. Gaiotto, A. Kapustin, N. Seiberg and B. Willett, Generalized Global Symmetries, JHEP 02 (2015) 172 [arXiv: 1412.5148] [INSPIRE].

[16] X.-G. Wen, Emergent anomalous higher symmetries from topological order and from dynamical electromagnetic field in condensed matter systems, Phys. Rev. B 99 (2019) 205139 [arXiv: 1812.02517] [INSPIRE].

[17] S. Chatterjee, S. Sachdev and M. Scheurer, Intertwining topological order and broken symmetry in a theory of fluctuating spin density waves, Phys. Rev. Lett. 119 (2017) 227002 [arXiv: 1705.06289] [INSPIRE]. 
[18] S. Sachdev and S. Chatterjee, Insulators and metals with topological order and discrete symmetry breaking, Phys. Rev. B 95 (2017) 205133 [arXiv: 1703.00014] [INSPIRE].

[19] M.S. Scheurer, S. Chatterjee, W. Wu, M. Ferrero, A. Georges and S. Sachdev, Topological order in the pseudogap metal, Proc. Nat. Acad. Sci. 115 (2018) E3665 [arXiv:1711.09925] [INSPIRE].

[20] S. Sachdev, H.D. Scammell, M.S. Scheurer and G. Tarnopolsky, Gauge theory for the cuprates near optimal doping, Phys. Rev. B 99 (2019) 054516 [arXiv:1811.04930] [INSPIRE].

[21] M.A. Stephanov, QCD phase diagram and the critical point, Prog. Theor. Phys. Suppl. 153 (2004) 139 [hep-ph/0402115] [INSPIRE].

[22] K. Fukushima and T. Hatsuda, The phase diagram of dense QCD, Rept. Prog. Phys. 74 (2011) 014001 [arXiv: 1005.4814] [INSPIRE].

[23] H.-c. Ren, Color superconductivity of QCD at high baryon density, 2004, hep-ph/0404074 [INSPIRE].

[24] R. Casalbuoni, Lecture Notes on Superconductivity: Condensed Matter and QCD, arXiv: 1810.11125 [INSPIRE].

[25] B.C. Barrois, Superconducting Quark Matter, Nucl. Phys. B 129 (1977) 390 [INSPIRE].

[26] D. Bailin and A. Love, Superfluidity and Superconductivity in Relativistic Fermion Systems, Phys. Rept. 107 (1984) 325 [INSPIRE].

[27] M.G. Alford, K. Rajagopal and F. Wilczek, Color flavor locking and chiral symmetry breaking in high density QCD, Nucl. Phys. B 537 (1999) 443 [hep-ph/9804403] [INSPIRE].

[28] T. Schäfer and F. Wilczek, Continuity of quark and hadron matter, Phys. Rev. Lett. 82 (1999) 3956 [hep-ph/9811473] [INSPIRE].

[29] M.G. Alford, A. Schmitt, K. Rajagopal and T. Schäfer, Color superconductivity in dense quark matter, Rev. Mod. Phys. 80 (2008) 1455 [arXiv:0709.4635] [INSPIRE].

[30] A.P. Balachandran, S. Digal and T. Matsuura, Semi-superfluid strings in high density QCD, Phys. Rev. D 73 (2006) 074009 [hep-ph/0509276] [INSPIRE].

[31] E. Nakano, M. Nitta and T. Matsuura, Non-Abelian strings in high density QCD: Zero modes and interactions, Phys. Rev. D 78 (2008) 045002 [arXiv:0708.4096] [InSPIRE].

[32] M. Eto, Y. Hirono, M. Nitta and S. Yasui, Vortices and Other Topological Solitons in Dense Quark Matter, PTEP 2014 (2014) 012D01 [arXiv: 1308.1535] [INSPIRE].

[33] A. Yamamoto, Non-Abelian vortex in lattice gauge theory, PTEP 2018 (2018) 103B03 [arXiv: 1804.08051] [INSPIRE].

[34] Y. Hirono and Y. Tanizaki, Quark-Hadron Continuity beyond the Ginzburg-Landau Paradigm, Phys. Rev. Lett. 122 (2019) 212001 [arXiv:1811.10608] [INSPIRE].

[35] A. Cherman, S. Sen and L.G. Yaffe, Anyonic particle-vortex statistics and the nature of dense quark matter, arXiv: 1808.04827 [INSPIRE].

[36] M.G. Alford, G. Baym, K. Fukushima, T. Hatsuda and M. Tachibana, Continuity of vortices from the hadronic to the color-flavor locked phase in dense matter, Phys. Rev. D 99 (2019) 036004 [arXiv: 1803.05115] [INSPIRE].

[37] C. Chatterjee, M. Nitta and S. Yasui, Quark-hadron continuity under rotation: Vortex continuity or boojum?, Phys. Rev. D 99 (2019) 034001 [arXiv:1806.09291] [INSPIRE]. 
[38] Y. Hirono, T. Kanazawa and M. Nitta, Topological Interactions of Non-Abelian Vortices with Quasi-Particles in High Density QCD, Phys. Rev. D 83 (2011) 085018 [arXiv: 1012.6042] [INSPIRE].

[39] M. Bergeron, G.W. Semenoff and R.J. Szabo, Canonical bf type topological field theory and fractional statistics of strings, Nucl. Phys. B 437 (1995) 695 [hep-th/9407020] [INSPIRE].

[40] G.Y. Cho and J.E. Moore, Topological BF field theory description of topological insulators, Annals Phys. 326 (2011) 1515 [arXiv: 1011.3485] [INSPIRE].

[41] P. Putrov, J. Wang and S.-T. Yau, Braiding Statistics and Link Invariants of Bosonic/Fermionic Topological Quantum Matter in $2+1$ and $3+1$ dimensions, Annals Phys. 384 (2017) 254 [arXiv: 1612.09298] [InSPIRE].

[42] X. Chen, A. Tiwari and S. Ryu, Bulk-boundary correspondence in $(3+1)$-dimensional topological phases, Phys. Rev. B 94 (2016) 045113 [arXiv: 1509.04266] [INSPIRE].

[43] G. 't Hooft, Naturalness, chiral symmetry, and spontaneous chiral symmetry breaking, NATO Sci. Ser. B 59 (1980) 135 [INSPIRE].

[44] T. Banks and N. Seiberg, Symmetries and Strings in Field Theory and Gravity, Phys. Rev. D 83 (2011) 084019 [arXiv: 1011.5120] [INSPIRE].

[45] A. Kapustin and N. Seiberg, Coupling a QFT to a TQFT and Duality, JHEP 04 (2014) 001 [arXiv:1401.0740] [INSPIRE].

[46] A. Tiwari, X. Chen and S. Ryu, Wilson operator algebras and ground states of coupled BF theories, Phys. Rev. B 95 (2017) 245124 [arXiv:1603.08429] [InSPIRE].

[47] E. Lake, Higher-form symmetries and spontaneous symmetry breaking, arXiv:1802.07747 [INSPIRE].

[48] X.-G. Wen, Classifying gauge anomalies through symmetry-protected trivial orders and classifying gravitational anomalies through topological orders, Phys. Rev. D 88 (2013) 045013 [arXiv: 1303.1803] [INSPIRE].

[49] A. Kapustin and R. Thorngren, Anomalies of discrete symmetries in various dimensions and group cohomology, arXiv:1404.3230 [INSPIRE].

[50] J.C. Wang, Z.-C. Gu and X.-G. Wen, Field theory representation of gauge-gravity symmetry-protected topological invariants, group cohomology and beyond, Phys. Rev. Lett. 114 (2015) 031601 [arXiv: 1405.7689] [INSPIRE].

[51] E. Witten, The "Parity" Anomaly On An Unorientable Manifold, Phys. Rev. B 94 (2016) 195150 [arXiv: 1605.02391] [INSPIRE].

[52] Y. Tachikawa and K. Yonekura, On time-reversal anomaly of $2+1 d$ topological phases, PTEP 2017 (2017) 033B04 [arXiv: 1610.07010] [INSPIRE].

[53] D. Gaiotto, A. Kapustin, Z. Komargodski and N. Seiberg, Theta, Time Reversal and Temperature, JHEP 05 (2017) 091 [arXiv: 1703.00501] [INSPIRE].

[54] Y. Tanizaki and Y. Kikuchi, Vacuum structure of bifundamental gauge theories at finite topological angles, JHEP 06 (2017) 102 [arXiv:1705.01949] [INSPIRE].

[55] Z. Komargodski, A. Sharon, R. Thorngren and X. Zhou, Comments on Abelian Higgs Models and Persistent Order, SciPost Phys. 6 (2019) 003 [arXiv: 1705.04786] [InSPIRE].

[56] Z. Komargodski, T. Sulejmanpasic and M. Ünsal, Walls, anomalies and deconfinement in quantum antiferromagnets, Phys. Rev. B 97 (2018) 054418 [arXiv:1706.05731] [InSPIRE]. 
[57] H. Shimizu and K. Yonekura, Anomaly constraints on deconfinement and chiral phase transition, Phys. Rev. D 97 (2018) 105011 [arXiv:1706.06104] [INSPIRE].

[58] J. Wang, X.-G. Wen and E. Witten, Symmetric Gapped Interfaces of SPT and SET States: Systematic Constructions, Phys. Rev. X 8 (2018) 031048 [arXiv:1705.06728] [InSPIRE].

[59] D. Gaiotto, Z. Komargodski and N. Seiberg, Time-reversal breaking in $Q C D_{4}$, walls and dualities in $2+1$ dimensions, JHEP 01 (2018) 110 [arXiv:1708.06806] [INSPIRE].

[60] Y. Tanizaki, T. Misumi and N. Sakai, Circle compactification and 't Hooft anomaly, JHEP 12 (2017) 056 [arXiv:1710.08923] [INSPIRE].

[61] Y. Tanizaki, Y. Kikuchi, T. Misumi and N. Sakai, Anomaly matching for the phase diagram of massless $\mathbb{Z}_{N}-Q C D$, Phys. Rev. D 97 (2018) 054012 [arXiv: 1711.10487] [InSPIRE].

[62] M. Guo, P. Putrov and J. Wang, Time reversal, SU(N) Yang-Mills and cobordisms: Interacting topological superconductors/insulators and quantum spin liquids in $3+1 D$, Annals Phys. 394 (2018) 244 [arXiv:1711.11587] [InSPIRE].

[63] T. Sulejmanpasic and Y. Tanizaki, C-P-T anomaly matching in bosonic quantum field theory and spin chains, Phys. Rev. B 97 (2018) 144201 [arXiv:1802.02153] [INSPIRE].

[64] Y. Tanizaki and T. Sulejmanpasic, Anomaly and global inconsistency matching: $\theta$-angles, $\mathrm{SU}(3) / \mathrm{U}(1)^{2}$ nonlinear $\sigma$-model, $\mathrm{SU}(3)$ chains and its generalizations, Phys. Rev. B 98 (2018) 115126 [arXiv:1805.11423] [INSPIRE].

[65] C. Córdova and T.T. Dumitrescu, Candidate Phases for SU(2) Adjoint QCD 4 with Two Flavors from $\mathcal{N}=2$ Supersymmetric Yang-Mills Theory, arXiv:1806.09592 [INSPIRE].

[66] M.M. Anber and E. Poppitz, Two-flavor adjoint QCD, Phys. Rev. D 98 (2018) 034026 [arXiv: 1805.12290] [INSPIRE].

[67] M.M. Anber and E. Poppitz, Anomaly matching, (axial) Schwinger models and high-T super Yang-Mills domain walls, JHEP 09 (2018) 076 [arXiv: 1807.00093] [INSPIRE].

[68] Y. Tanizaki, Anomaly constraint on massless $Q C D$ and the role of Skyrmions in chiral symmetry breaking, JHEP 08 (2018) 171 [arXiv:1807.07666] [INSPIRE].

[69] K. Yonekura, Anomaly matching in QCD thermal phase transition, JHEP 05 (2019) 062 [arXiv: 1901.08188] [INSPIRE].

[70] C.G. Callan Jr. and J.A. Harvey, Anomalies and Fermion Zero Modes on Strings and Domain Walls, Nucl. Phys. B 250 (1985) 427 [inSPIRE].

[71] Y. Hidaka, Y. Hirono, M. Nitta, Y. Tanizaki and R. Yokokura, Topological order in the color-flavor locked phase of $(3+1)$-dimensional $\mathrm{U}(N)$ gauge-Higgs system, arXiv: 1903.06389 [INSPIRE]. 